\title{
Synthesis of a fucosylated and a non-fucosylated core structure of xylose-containing carbohydrate chains from $\mathrm{N}$-glycoproteins
}

\author{
Jos G.M. van der Ven 1,a , János Kerékgyártó b, \\ Johannis P. Kamerling a,*, András Lipták ${ }^{\text {b }}$, \\ Johannes F.G. Vliegenthart ${ }^{\text {a }}$ \\ a Bijvot Center, Department of Bio-Organic Chemistry, Utrecht University, P.O. Box 80.075, \\ NL-3508 TB Utrecht, Netherlands \\ ${ }^{b}$ Institute of Biochemistry, L. Kossuth University, P.O. Box 55, H-401O Debrecen, Iungary
}

Received 14 March 1994; accepted 16 May 1994

\begin{abstract}
The synthesis is reported of methyl 2-acetamido-4-O-[2-acetamido-2-deoxy-4-O-(3,6-di- $O$ - $\alpha$-Dmannopyranosyl-2- $O$ - $\beta$-D-xylopyranosyl- $\beta$-D-mannopyranosyl) - $\beta$-D-glucopyranosyl ] -2-deoxy- $\beta$ D-glucopyranoside (4) and methyl 2-acetamido-4- $O$-[2-acetamido-2-deoxy-4- $O$-(3,6-di- $O$ - $\alpha$-Dmannopyranosyl-2- $O-\beta$-1)-xylopyranosyl- $\beta$-D-mannopyranosyl) $-\beta$-1-glucopyranosyl]-2-deoxy-6$O$ - $\alpha$-L-fucopyranosyl- $\beta$-D-glucopyranoside (5), which represent the invariant hexasaccharide core structure of the xylose-containing glycans of $\mathrm{N}$-glycoproteins and its 6-O-fucosylated derivative. Ethyl 4-O-[3-O-allyl-4- $O$-benzoyl-6- $O$-tert-butyldimethylsilyl-2- $O$ - $(2,3,4$-tri- $O$-acetyl- $\beta$-D-xylopyranosyl )- $\beta$-D-mannopyranosyl ]-3,6-di- $O$-benzyl-2-deoxy-2-phthalimido-1-thio- $\beta$-D-glucopyranoside (9) was coupled with methyl 3,6-di- $O$-benzyl-2-deoxy-2-phthalimido- $\beta$-D-glucopyranoside (11). Desilylation of the resulting tetrasaccharide derivative, followed by condensation with $2,3,4,6-$ tetra- $O$-acetyl- $\alpha$-D-mannopyranosyl trichloroacetimidate (7), gave methyl 4-O- $\{4-O$ - [3-O-allyl-4$O$-benzoyl-6-O-(2,3,4,6-tetra- $O$-acetyl- $\alpha$-D-mannopyranosyl) - $2-O$ - $(2,3,4$-tri- $O$-acetyl- $\beta$-D-xylopyranosyl ) - $\beta$-D-mannopyranosyl ] -3,6-di- $O$-benzyl-2-deoxy-2-phthalimido- $\beta$-D-glucopyranosyl $\}$ 3,6-di- $O$-benzyl-2-deoxy-2-phthalimido- $\beta$-D-glucopyranoside (14). Deallylation of 14, followed by condensation with 7 and deprotection, gave hexasaccharide 4. Ethyl 3,6-di- $O$-benzyl-2-deoxy-4-O[4,6-di- $O$-acetyl-3- $O$-allyl-2- $O$ - $(2,3,4$-tri- $O$-acetyl- $\beta$-D-xylopyranosyl $)-\beta$-D-mannopyranosyl]-2phthalimido-1-thio- $\beta$-D-glucopyranoside (17) was coupled with methyl 3- $O$-benzyl-2-deoxy-6- $O$ (4-methoxybenzyl) -2-phthalimido- $\beta$-D-glucopyranoside. Demethoxybenzylation of the tetrasaccharide derivative thus obtained, followed by fucosylation using ethyl 2,3,4-tri-O-benzyl-1-thio-

\footnotetext{
* Corresponding author.

${ }^{1}$ Present address: Department of Biochemistry, University of Groningen, Nijenborgh 4, NL-9747 AG Groningen, Netherlands.
} 
$\beta$-L-fucopyranoside, gave methyl 3-O-benzyl-2-deoxy-4-O-\{3,6-di- $O$-benzyl-2-deoxy-4- $O$ - $[4,6-$ di- $O$-acetyl-3- $O$-allyl-2- $O$-( $2,3,4-$ tri- $O$-acetyl- $\beta$-D-xylopyranosyl $)$ - $\beta$-D-mannopyranosyl ] 2 -phthalimido- $\beta$-D-glucopyranosyl $\}-2$-phthalimido-6- $O$-( $2,3,4$-tri- $O$-benzyl- $\alpha$-L-fucopyranosyl) $-\beta$-D-glucopyranoside (23). $O$-Deacetylation followed by tert-butyldimethylsilylation, benzoylation, and desi-

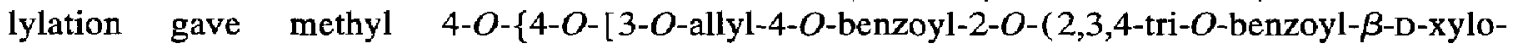
pyranosyl) - $\beta$-D-mannopyranosyl]-3,6-di- $O$-benzyl-2-deoxy-2-phthalimido- $\beta$-D-glucopyranosyl $\}$-3$O$-benzyl-2-deoxy-2-phthalimido-6- $O$ - $(2,3,4$-tri- $O$-benzyl- $\alpha$-L-fucopyranosyl $)$ - $\beta$-D-glucopyranoside (24). Mannosylation of 24 using 7, followed by deallylation, further mannosylation with 7, and deprotection, gave the heptasaccharide $\mathbf{5}$.

Keywords: Hemocyanin; Lectin; Xylose-type N-glycan; Glycoprotein; Oligosaccharide synthesis

\section{Introduction}

Since their discovery in the late 70 's, xylose-containing N-linked carbohydrate chains have become well established constituents of glycoproteins, mostly of plant but also of animal origin [1]. Because $\beta$-D-Xylp is always $(1 \rightarrow 2)$-linked to $\beta$-D-Man $p$ of the usual $\mathrm{Man}_{3} \mathrm{GlcNAc}_{2}$ core structure, a new invariant hexasaccharide core structure $(1)$ can be defined for the xylose-type $\mathrm{N}$-linked glycans. Already many glycans based on this core structure have been isolated and characterized [1]. A striking difference in the xylosecontaining $\mathrm{N}$-glycans of plant and animal origin is found in the fucosylation (if present) of the innermost Glc $p$ NAc residue of the core structure. In xylose-type glycans of animal origin, L-Fucp is $\alpha-(1 \rightarrow 6)$-linked, as in the glycans derived from $\alpha$-hemocyanin of Helix pomatia [2] (2), and in those of plant origin, such as a seed lectin obtained from Sophora japonica [3,4] (3), it is $\alpha-(1 \rightarrow 3)$-linked.

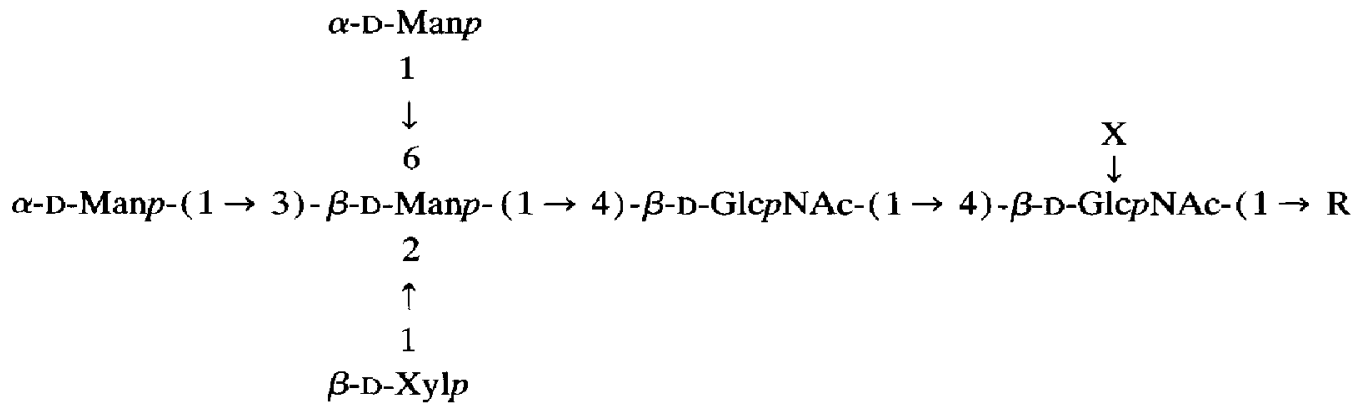

$1 \mathrm{X}=\mathrm{H}, \mathrm{R}=\mathrm{Asn}$

$2 \mathrm{X}=\alpha$-L-Fucp- $(1 \rightarrow 6), \mathrm{R}=$ Asn

$3 \mathrm{X}=\alpha$-L-Fucp- $(1 \rightarrow 3), \mathrm{R}=$ Asn

$4 \mathrm{X}=\mathrm{H}, \mathrm{R}=\mathrm{OMe}$

$5 \mathrm{X}=\alpha$-L-Fucp- $(1 \rightarrow 6), \mathrm{R}=\mathrm{OMe}$

As part of our program focused on the structure elucidation [1], conformation analysis [5-7], organic synthesis, and biosynthesis [8] of xylose-containing glycans, the synthesis of structural elements, comprising only the branching point of the core structure, has been reported [9-11]. We now describe the synthesis of the hexasaccharide methyl $\beta$-glycoside 

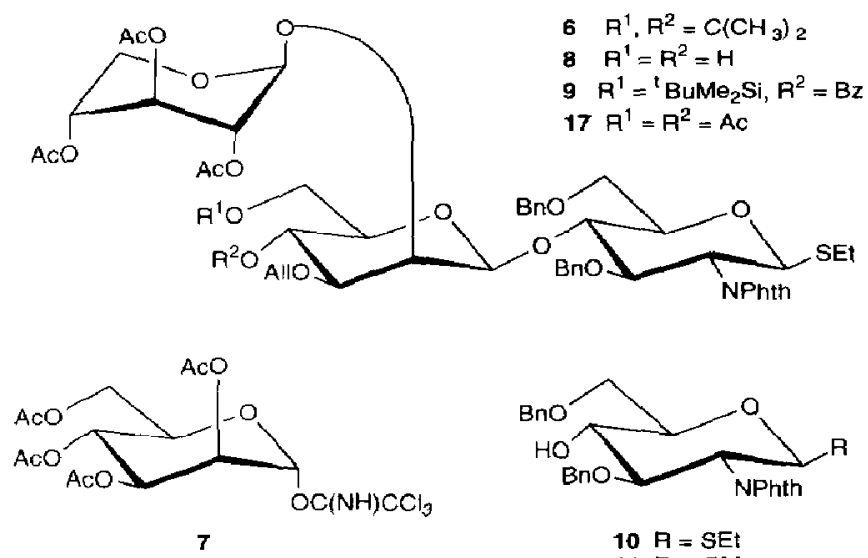

$$
\begin{aligned}
& 10 R=S E k \\
& 11 R=\text { OMe }
\end{aligned}
$$
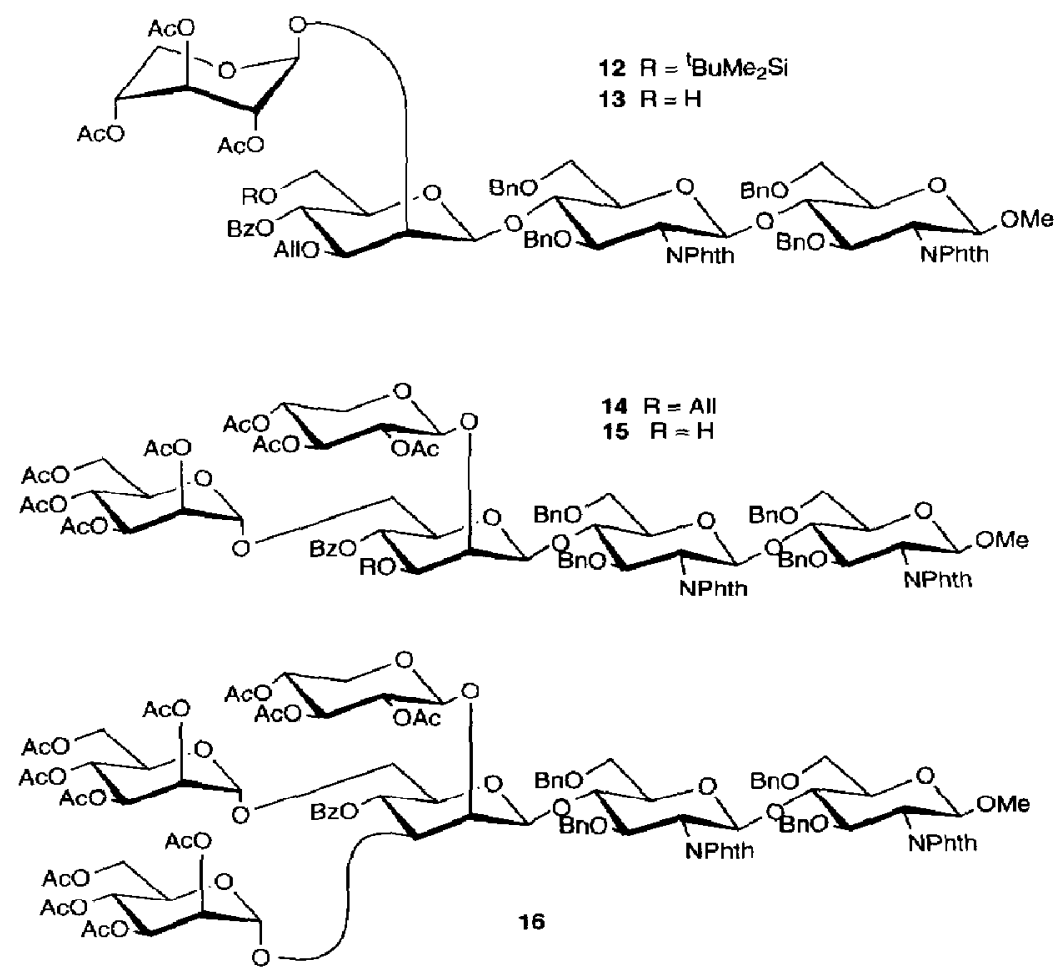

4 , representing the complete invariant core structure, and the heptasaccharide methyl $\beta$ glycoside 5, having a Fuc residue $\alpha-(1 \rightarrow 6)$-linked to the innermost GlcNAc residue.

\section{Results and discussion}

Previously, the synthesis of the selectively protected trisaccharide ethyl 4-O-[3-O-allyl4,6- $O$-isopropylidene-2- $O$ - $(2,3,4$-tri- $O$-acetyl- $\beta$-D-xylopyranosyl $)-\beta$-D-mannopyrano- 

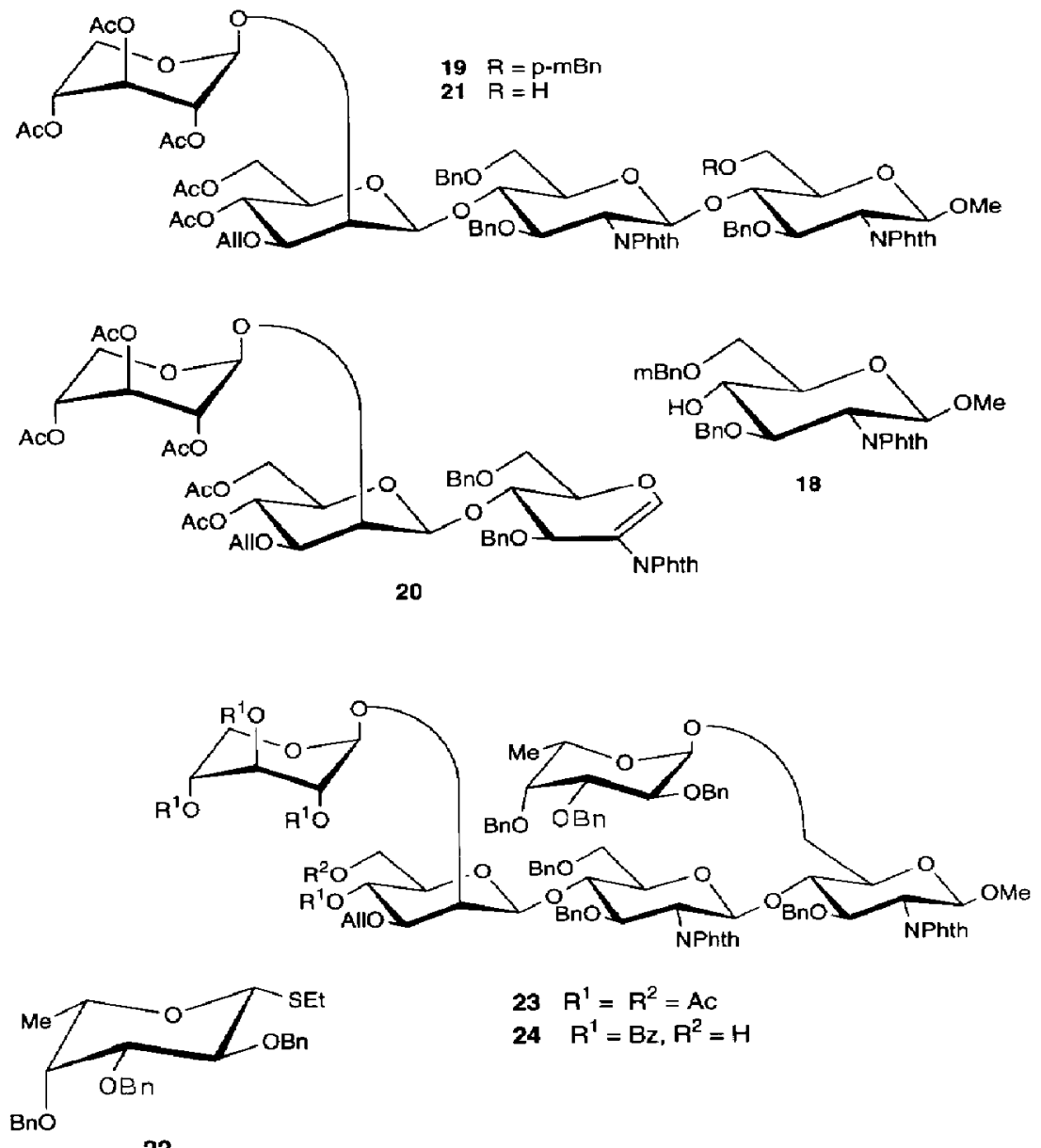

22

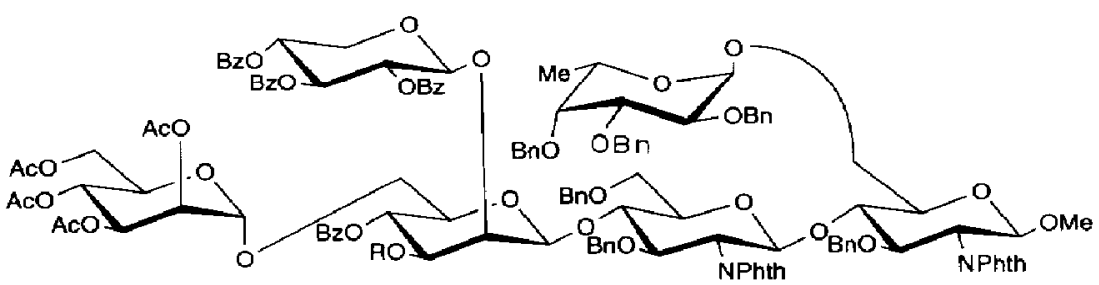

25 月 $=$ Allyl

$26 \mathrm{~B}=\mathrm{H}$

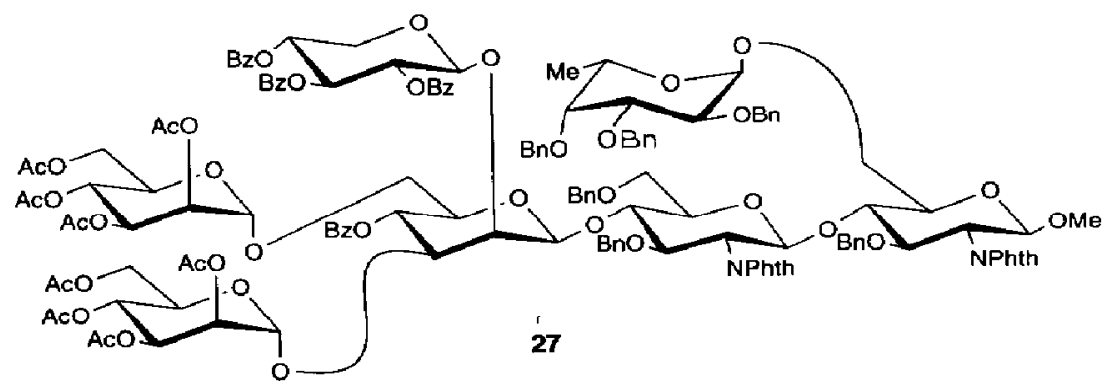


syl]-3,6-di- $O$-benzyl-2-deoxy-2-phthalimido-1-thio- $\beta$-D-glucopyranoside $[10]$ (6) has been reported. This unit was chosen as the central building block for the synthesis of the glycans 4-5. The presence of the isopropylidene acetal at $\mathrm{HO}-4^{\prime}, 6^{\prime}$ and the allyl group at HO-3' gives the opportunity to carry out regioselective $\alpha$-mannosylations at $\mathrm{HO}-6^{\prime}$ and/or HO-3'. Furthermore, by activating the thioethyl glycoside, this building block (or extended with $\alpha$-D-Manp residues) can serve as a glycosyl donor. Coupling of this donor to two different acceptors, namely, derivatized glucosamine or $\alpha-(1 \rightarrow 6)$-fucosylated glucosamine, looks attractive for the synthesis of the two desired glycans.

However, the regioselective coupling of mannosyl donors, such as 2,3,4,6-tetra- $O$-acetyl$\alpha$-D-mannopyranosyl trichloroacetimidate [9] (7) and 2,3,4,6-tetra- $O$-acetyl- $\alpha$-Dmannopyranosyl bromide, with deisopropylidenated $6(8)$ turned out to be extremely difficult. The main problem was the relative lability of the thioethyl group of the acceptor during coupling reactions, giving rise to degradation of the acceptor. Moreover, the transfer of the thioethyl group to the anomeric center of the donor (trans-glycosidation) was sometimes observed. As we had already noticed during the synthesis of $6[10]$, it was only possible to carry out coupling reactions employing glycosyl bromides catalyzed by silver triflate in toluene-dichloromethane at $-40^{\circ} \mathrm{C} /-60^{\circ} \mathrm{C}$. Although some interesting protected tetra- and penta-saccharides [12] could be prepared using this glycosylation procedure, the yields were extremely low and undesired ortho-ester formation was also encountered. Thus, although the thioethyl glycoside behaved well during all kinds of protective group manipulation, for example, $O$-deacetylation, deisopropylidenation, oxidation-reduction, and deallylation [10], its behaviour in glycosylation reactions was not satisfactory. It must be noted that this behaviour might well be due to the use of a 2-deoxy-2-phthalimidothioglycoside, because many other thioglycosides have been described which were stable during coupling reactions $[13,14]$.

To build up the oligosaccharides 4-5, it was decided that it would be better to couple the trisaccharide donor first to an acceptor, and then to elongate the resulting structures in a stepwise manner with mannosyl donors at the non-reducing end. This is now described separately for the two target structures 4 and 5 , in both cases starting with compound 8.

Synthesis of hexasaccharide 4.-Ethyl 4-O-[3-O-allyl-2-O-(2,3,4-tri-O-acetyl- $\beta$-Dxylopyranosyl)- $\beta$-D-mannopyranosyl] -3,6-di- $O$-benzyl-2-deoxy-2-phthalimido-1-thio- $\beta$ D-glucopyranoside [10] (8) was silylated on $\mathrm{HO}-6^{\prime}$ with tert-butyldimethylsilyl chloride

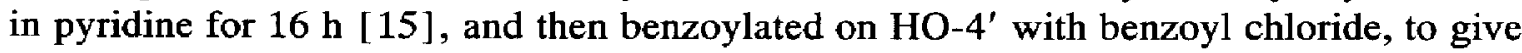
the trisaccharide donor 9 (93\%). The choice of the protective groups of HO-3', $4^{\prime}, 6^{\prime}$ allows the possibility to perform the planned regioselective $\alpha$-mannosylations in a later stage. Ethyl 3,6-di- $O$-benzyl-2-deoxy-2-phthalimido-1-thio- $\beta$-D-glucopyranoside [10] (10) was converted into the corresponding methyl glycoside $11(66 \%)$ by treatment with methanol and methyl triflate in toluene. Coupling of 9 with 11, using $N$-iodosuccinimide (NIS) and trifluoromethanesulfonic acid [14] (HOTf) as catalyst, gave the tetrasaccharide derivative $12(52 \%)$. Removal of the tert-butyldimethylsilyl group, using $p$-toluenesulfonic acid in acetonitrile-water, gave the tetrasaccharide acceptor $13(97 \%)$. Mannosylation of 13 with 2,3,4,6-tetra- $O$-acetyl- $\alpha$-D-mannopyranosyl trichloroacetimidate [9] (7) in dichloromethane at $-20^{\circ} \mathrm{C}$, using trimethylsilyl triflate as catalyst, gave $14(71 \%)$. After deallylation of 14 using the Wilkinson catalyst in the presence of 1,4-diazabicyclo[2.2.2] octane [16] 
followed by hydrolysis ( $\rightarrow 15,85 \%$ ), mannosylation with 7 , as described for 13 , gave the hexasaccharide derivative $16(59 \%)$. The chosen procedure for the deprotection of 16, involving dephthaloylation/deacylation with methylamine in ethanol [17], re- $N$-acetylation with acetic anhydride in methanol, and then hydrogenolysis using palladium on activated carbon, was not completely successful. ${ }^{1} \mathrm{H}$ NMR analysis of the final product showed an incomplete dephthaloylation, and therefore the residue was treated with hydrazine acetate in boiling ethanol [18], followed by re- $N$-acetylation, column chromatography, and RPHPLC, to give the hexasaccharide $4(77 \%)$. For ${ }^{1} \mathrm{H}$ NMR data, see Table 1.

Synthesis of heptasaccharide 5.-Ethyl 3,6-di- $O$-benzyl-2-deoxy-4-O-[4,6-di- $O$-acetyl3- $O$-allyl-2- $O$-( $2,3,4$-tri- $O$-acetyl- $\beta$-D-xylopyranosyl)- $\beta$-D-mannopyranosyl $]$-2-phthalimido-1-thio- $\beta$-D-glucopyranoside (17) was prepared from 8 via acetylation with pyridineacetic anhydride (99\%). The choice of this trisaccharide donor also makes regioselective $\alpha$-mannosylation possible in a later stage. Condensation of 17 with methyl 3-O-benzyl-2deoxy-6- $O$-(4-methoxybenzyl)-2-phthalimido- $\beta$-D-glucopyranoside $[18]$ (18), using NIS/HOTf, gave the tetrasaccharide derivative $19(61 \%)$ and the trisaccharide derivative 20 as the major side-product $[19,20](20 \%)$. Demethoxybenzylation of 19, using ceric (IV) ammonium nitrate in acetonitrile-water [21], yielded the tetrasaccharide acceptor 21 ( $86 \%$ ), which was fucosylated with ethyl 2,3,4-tri- $O$-benzyl-1-thio- $\beta$-L-fucopyranoside [19] (22), in dichloroethane-ether using iodonium dicollidine perchlorate [14] as promoter, to give the pentasaccharide derivative 23 (49\%) and an unknown compound 23-a.

Zcmplén deacetylation of 23 , followed by silylation of $\mathrm{HO}-6^{\prime \prime}$ with tert-butyldimethylsilyl chloride in pyridine [15], benzoylation of HO- $4^{\prime \prime \prime}, 2^{\prime \prime \prime \prime}, 3^{\prime \prime \prime \prime}, 4^{\prime \prime \prime \prime}$ using benzoyl chloride, and removal of the silyl ether with $p$-toluenesulfonic acid in acetonitrilewater gave methyl 4- $O-\{4-O-[3-O$-allyl-4- $O$-benzoyl-2- $O$ - $(2,3,4-$ tri- $O$-benzoyl- $\beta$-Dxylopyranosy1) - $\beta$-D-mannopyranosyl] -3,6-di- $O$-benzyl-2-deoxy-2-phthalimido- $\beta$-D-glucopyranosyl $\}-3-O$-benzyl-2-deoxy-2-phthalimido-6-O-( 2,3,4-tri- $O$-benzyl- $\alpha$-L-fucopyranosy1)- $\beta$-D-glucopyranoside $(24,72 \%)$. Mannosylation of 24 with imidate 7 in dichloromethane at $-20^{\circ} \mathrm{C}$, using trimethylsilyl triflate as catalyst, gave the hexasaccharide derivative $25(45 \%)$. Deallylation of 25 using the Wilkinson catalyst in the presence of 1,4-diazabicyclo[2.2.2] octane [16], followed by hydrolysis ( $\rightarrow 26,73 \%$ ), and subsequent mannosylation, as described for $\mathbf{2 4}$, gave the heptasaccharide derivative $27(88 \%)$. The chosen procedure for the deprotection of 27, involving dephthaloylation/deacylation using hydrazine acetate in boiling ethanol [18], followed by re- $N, O$-acetylation using pyridineacetic anhydride, and finally hydrogenolysis using palladium on carbon, was not completely successful. Again, ${ }^{1}$ H NMR analysis of the final product showed incomplete dephthaloylation, and it was therefore treated with methylamine in ethanol [17], followed by re- $N$ acetylation, column chromatography, and RP-HPLC, to afford the heptasaccharide 5 (83\%). For ${ }^{1} \mathrm{H}$ NMR data, see Table 1.

As was observed during the preparation of smaller oligosaccharides $[10,11]$, the ${ }^{1} \mathbf{H}$ NMR data of the compounds containing a $\beta$-D-xylopyranose residue showed that the ${ }^{3} J-$ values of the skeleton protons of xylose vary to some extent. As has been discussed [10,11], this behaviour can be ascribed to the presence of a ${ }^{1} C_{4} \rightleftarrows{ }^{4} C_{1}$ conformational equilibrium, but also distortions of the ${ }^{4} C_{1}$ chair can lead to deviating $J$-values. At least for $9,12,13,17$, $19,20,21,23$, and 24 , the D-xylose residue does not seem to occur exclusively in the ${ }^{4} C_{1}$ chair conformation $\left({ }^{3} J_{1,2} \leq 3.5 \mathrm{~Hz}\right)$. 
Table 1

500-MHz ${ }^{1} \mathrm{H}$ NMR data of hexasaccharide 4 and heptasaccharide 5, and of relevant reference compounds

\begin{tabular}{|c|c|c|c|c|c|c|c|}
\hline \multirow[t]{4}{*}{ Residue } & \multirow{4}{*}{$\begin{array}{l}\text { Reporter group } \\
(J)\end{array}$} & \multicolumn{6}{|c|}{$\delta(\mathrm{ppm}) / J(\mathrm{~Hz})$} \\
\hline & & \multicolumn{3}{|l|}{$\mathbf{X}=\mathbf{H}$} & \multicolumn{3}{|c|}{$\mathrm{X}=\alpha-\mathrm{L}-\mathrm{Fuc} p-(1 \rightarrow 6)$} \\
\hline & & 4 & $1[22]$ & $\begin{array}{l}\text { Ref } \\
{[23]}\end{array}$ & 5 & 2 & $\operatorname{Ref}[1]$ \\
\hline & & $\mathrm{R}=\mathrm{OMe}$ & $\mathbf{R}=\mathbf{A s n}$ & $\mathrm{R}=\mathrm{OH}^{\mathrm{a}}$ & $\mathrm{R}=\mathrm{OMe}$ & $\mathbf{R}=\mathbf{A s n}$ & $\mathrm{R}=\mathrm{OH}^{\mathrm{a}}$ \\
\hline \multirow[t]{4}{*}{ GleNAc-1 } & $\mathrm{H}-1\left(J_{1,2}\right)$ & $4.432(8.1)$ & 5.048 & $5.189^{\alpha}$ & $4.429(8.3)$ & 5.088 & $5.181^{\alpha}$ \\
\hline & & & & $4.703^{\beta}$ & & & $4.694^{\beta}$ \\
\hline & NAc & 2.029 & 2.010 & 2.039 & 2.029 & 2.010 & 2.040 \\
\hline & $\mathrm{OMe}$ & 3.495 & & & 3.487 & & \\
\hline \multirow[t]{4}{*}{ GlcNAc-2 } & $\mathbf{H}-1\left(J_{1,2}\right)$ & $4.597(8.0)$ & 4.608 & $4.615^{\alpha}$ & $4.670(8.0)$ & 4.686 & $4.666^{\alpha}$ \\
\hline & & & & $4.607^{\beta}$ & & & $4.673^{B}$ \\
\hline & NAc & 2.077 & 2.070 & 2.074 & 2.087 & 2.086 & $2.088^{\alpha}$ \\
\hline & & & & & & & $2.085^{\beta}$ \\
\hline \multirow[t]{2}{*}{ Man-3 } & $\mathrm{H}-1$ & 4.869 & 4.869 & 4.875 & 4.871 & 4.872 & 4.873 \\
\hline & $\mathrm{H}-2\left(I_{2,3}\right)$ & $4.265(3.1)$ & 4.264 & 4.265 & $4.265(3.2)$ & 4.267 & 4.266 \\
\hline \multirow[t]{2}{*}{ Man-4 } & $\mathrm{H}-1\left(J_{1,2}\right)$ & $5.121(1.7)$ & 5.121 & 5.122 & $5.122(1.7)$ & 5.122 & 5.123 \\
\hline & $\mathrm{H}-2$ & 4.038 & 4.037 & 4.041 & 4.038 & 4.037 & 4.038 \\
\hline \multirow[t]{2}{*}{ Man-4' } & $\mathrm{H}-1\left(J_{1,2}\right)$ & $4.913(1.8)$ & 4.911 & 4.912 & $4,913(1.8)$ & 4.914 & 4.913 \\
\hline & $\mathrm{H}-2$ & 3.982 & 3.981 & 3.982 & 3.980 & 3.979 & 3.981 \\
\hline \multirow[t]{5}{*}{ Xyl } & $\mathrm{H}-1\left(J_{1.2}\right)$ & $4.448(7.7)$ & 4.447 & 4.453 & $4.450(7.7)$ & 4.449 & 4.450 \\
\hline & $\mathrm{H}-2\left(J_{2,3}\right)$ & $3.374(9.4)$ & 3.373 & 3.375 & $3.374(9.4)$ & 3.377 & 3.375 \\
\hline & $\mathrm{H}-3\left(J_{3,4}\right)$ & $3.440(9.3)$ & 3.438 & 3.444 & $3.456(9.3)$ & n.d. ${ }^{\text {b }}$ & 3.456 \\
\hline & H-5ax $\left(J_{4,5 a t}\right)$ & $3.248(10.6 /$ & 3.248 & 3.253 & $3.252(10.7 /$ & 3.252 & 3.253 \\
\hline & $\left.J_{5 a x, 5 \cdot q}\right)$ & $-11.7)$ & & & $-11.6)$ & & \\
\hline \multirow[t]{5}{*}{ Fuc } & $\mathrm{H}-1\left(J_{1,2}\right)$ & & & & $4.906(4.5)$ & 4.878 & $4.890^{\alpha}$ \\
\hline & & & & & & & $4.898^{\beta}$ \\
\hline & $\mathrm{H}-5$ & & & & 4.134 & n.d. ${ }^{b}$ & 4.100 \\
\hline & $\mathrm{H}-6\left(J_{5,6}\right)$ & & & & $1.229(6.7)$ & 1.206 & $1.210^{\alpha}$ \\
\hline & & & & & & & $1.222^{\beta}$ \\
\hline
\end{tabular}

${ }^{a}$ Oligosaccharide occurs as an anomeric mixture; $\alpha$ and $\beta$ refer to GlcNAc-1.

${ }^{b}$ n.d., Not determined.

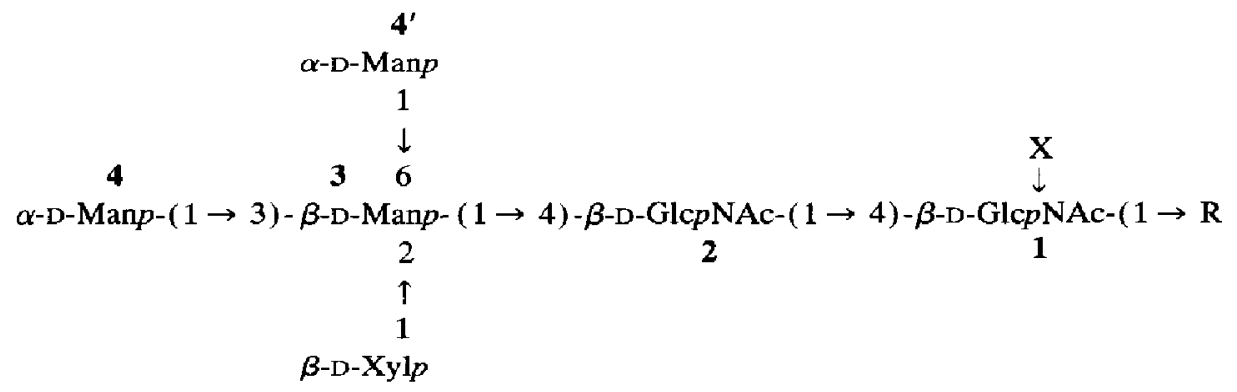

\section{Experimental}

General methods.-The ${ }^{1} \mathrm{H}$ ( 300 and $500 \mathrm{MHz}$ ) and ${ }^{13} \mathrm{C}$ (APT, 50 and $75 \mathrm{MHz}$ ) NMR spectra were recorded at $25^{\circ} \mathrm{C}$ with a Bruker AC 300, AM 500, or WP 200 spectrometer. 
Chemical shifts $(\delta)$ are given in ppm relative to the signal for internal $\mathrm{Me}_{4} \mathrm{Si}\left(\mathrm{CDCl}_{3}\right)$ or sodium 4,4-dimethyl-4-silapentane-1-sulfonate $\left(\mathrm{D}_{2} \mathrm{O}\right.$; indirectly to internal acetone $\delta$ $2.225)$ for ${ }^{1} \mathrm{H}$, and to the signal for internal $\mathrm{Me}_{4} \mathrm{Si}\left(\mathrm{CDCl}_{3}\right.$; indirectly to $\left.\mathrm{CDCl}_{3}, \delta 76.9\right)$ or external $\mathrm{Me}_{4} \mathrm{Si}\left(\mathrm{D}_{2} \mathrm{O}\right.$; indirectly to internal acetone, $\delta 31.55$ ) for ${ }^{13} \mathrm{C}$. Column chromatography was performed on Kieselgel 60 (Merck, <230 mesh) and fractions were monitored by TLC on Kieselgel $60 \mathrm{~F}_{254}$ (Merck) by detection with UV light and then charring with $\mathrm{H}_{2} \mathrm{SO}_{4}$. Preparative TLC was performed on Kieselgel $60 \mathrm{~F}_{254}$ (Merck, schichtdicke 0.5 $\mathrm{mm}$ ). Optical rotations were measured for solutions in $\mathrm{CHCl}_{3}$, unless otherwise stated, at $20^{\circ} \mathrm{C}$ with a Perkin-Elmer 241 polarimeter, using a $10-\mathrm{cm} 1-\mathrm{mL}$ cell. In the work-up procedures, washings were carried out three times with appropriate quantities of water or aq $5 \% \mathrm{NaHCO}_{3}$ unless indicated otherwise. Solvents were evaporated under reduced pressurc at $40^{\circ} \mathrm{C}$ (bath). All solvents were distilled from the appropriate drying agents.

Ethyl 4-0-13-O-allyl-4-O-benzoyl-6-O-tert-butyldimethylsilyl-2-O-(2, 3, 4-tri-O-acetyl$\beta$-D-xylopyranosyl)- $\beta$-D-mannopyranosyl]-3,6-di-O-benzyl-2-deoxy-2-phthalimido-1thio- $\beta$-D-glucopyranoside (9).-A solution of ethyl 4-O-[3-O-allyl-2-O-(2,3,4-tri-Oacetyl- $\beta$-D-xylopyranosyl ) $\beta$-D-mannopyranosyl] -3,6-di- $O$-benzyl-2-deoxy-2-phthalimido-1-thio- $\beta$-D-glucopyranoside $[10](8 ; 954 \mathrm{mg}, 0.96 \mathrm{mmol})$ and tert-butyldimethylsilyl chloride ( $434 \mathrm{mg}, 2.88 \mathrm{mmol}$ ) in pyridine $(15 \mathrm{~mL}$ ) was stirred for $16 \mathrm{~h}$. TLC (1:1 hexane-EtOAc) then showed the silylation to be complete $\left(R_{f} 0.56\right)$, and benzoyl chloride ( $200 \mu \mathrm{L}, 1.72 \mathrm{mmol}$ ) was added. After stirring for $16 \mathrm{~h}$, the mixture was diluted with $\mathrm{CH}_{2} \mathrm{Cl}_{2}(500 \mathrm{~mL})$, washed with aq $5 \% \mathrm{NaHCO}_{3}(2 \times 50 \mathrm{~mL})$ and water, dried $\left(\mathrm{MgSO}_{4}\right)$, filtered, and concentrated. Column chromatography ( $95: 5 \mathrm{CH}_{2} \mathrm{Cl}_{2}$-acetone) of the residue gave 9, isolated as a white foam $(1.1 \mathrm{~g}, 93 \%) ;[\alpha]_{\mathrm{D}}-30^{\circ}(c 1) ; R_{f} 0.54$. NMR data $\left(\mathrm{CDCl}_{3}\right):{ }^{1} \mathrm{H}, \delta 8.02-6.72(\mathrm{~m}, 19 \mathrm{H}, 3 \mathrm{Ph}$ and Phth $), 5.687\left(\mathrm{~m}, 1 \mathrm{H}, \mathrm{H}_{2} \mathrm{C}=\mathrm{CHCH}_{2} \mathrm{O}\right)$, 5.338 (t, $\left.1 \mathrm{H}, J_{4^{\prime}, 5^{\prime}} 9.8 \mathrm{~Hz}, \mathrm{H}-4^{\prime}\right), 5.260$ (d, $1 \mathrm{H}, J_{1,2} 10.4 \mathrm{~Hz}, \mathrm{H}-1$ ), $5.192\left(\mathrm{~d}, 1 \mathrm{H}_{,} J_{1^{\prime \prime}, 2^{\prime \prime}}\right.$ $\left.1.8 \mathrm{~Hz}, \mathrm{H}-1^{\prime \prime}\right), 5.125$ and $5.028\left(2 \mathrm{~m}, 2 \mathrm{H}, \mathrm{H}_{2} \mathrm{C}=\mathrm{CHCH}_{2} \mathrm{O}\right), 4.976$ and 4.401 ( 2 d, each 1 $\left.\mathrm{H}, \mathrm{PhCH}_{2} \mathrm{O}\right), 4.774$ and 4.552 ( $2 \mathrm{~d}$, each $\left.1 \mathrm{H}, \mathrm{PhCH}_{2} \mathrm{O}\right), 4.753\left(\mathrm{~m}, 1 \mathrm{H}, \mathrm{H}-4^{\prime \prime}\right), 4.678(\mathrm{~s}$, $1 \mathrm{H}, \mathrm{H}-1^{\prime}$ ) , 4.618 (dd, $1 \mathrm{H}, J_{5^{\prime \prime} e q / a x, 4^{\prime \prime}} 2.5, J_{5^{\prime \prime} e q, 5^{\prime \prime} a x}-13.1 \mathrm{~Hz}, \mathrm{H}-5^{\prime \prime} e q$ or H-5"ax), 3.315 (dd, $\left.1 \mathrm{H}, J_{3^{\prime}, 2^{\prime}} 3.0, J_{3^{\prime}, 4^{\prime}} 9.9 \mathrm{~Hz}, \mathrm{H}-3^{\prime}\right), 2.646\left(\mathrm{~m}, 2 \mathrm{H}, \mathrm{CH}_{3} \mathrm{CH}_{2} \mathrm{~S}\right.$ ), 2.148 and 2.131 ( $2 \mathrm{~s}$, 3 and $6 \mathrm{H}, 3 \mathrm{Ac}), 1.180\left(\mathrm{t}, 3 \mathrm{H}, \mathrm{CH}_{3} \mathrm{CH}_{2} \mathrm{~S}\right), 0.718$ [s, $\left.9 \mathrm{H},\left(\mathrm{CH}_{3}\right)_{3} \mathrm{CSi}\right],-0.146$ and $-0.208\left[2 \mathrm{~s}\right.$, each $\left.3 \mathrm{H},\left(\mathrm{CH}_{3}\right)_{2} \mathrm{Si}\right] ;{ }^{13} \mathrm{C}, \delta 169.5,169.4$, and $168.5\left(3 \mathrm{COCH}_{3}\right), 167.6$ and 167.1 (CO Phth), 164.9 (COPh), 138.7, 137.8, 134.1, 133.3, 132.7, 131.4, 131.3, 129.9, 129.3, 128.2-127.3, 126.4, and $122.9\left(\mathrm{C}_{6} \mathrm{H}_{5} \mathrm{CH}_{2} \mathrm{O}, \mathrm{C}_{6} \mathrm{H}_{5} \mathrm{CO}, \mathrm{H}_{2} \mathrm{C}=\mathrm{CHCH}_{2} \mathrm{O}\right.$, and Phth), $116.6\left(\mathrm{H}_{2} \mathrm{C}=\mathrm{CHCH}_{2} \mathrm{O}\right), 101.1$ and $97.5\left(\mathrm{C}-1^{\prime}, 1^{\prime \prime}\right), 80.8,79.9,78.6,78.4,78.2,75.2,72.7$, $69.2,67.2,67.0$, and $66.1\left(\mathrm{C}-1,3,4,5,2^{\prime}, 3^{\prime}, 4^{\prime}, 5^{\prime}, 2^{\prime \prime}, 3^{\prime \prime}, 4^{\prime \prime}\right), 74.7,73.3,70.4,68.9,63.5$, and $58.3\left(\mathrm{C}-6,6^{\prime}, 5^{\prime \prime}, 2 \mathrm{PhCH}_{2} \mathrm{O}\right.$, and $\left.\mathrm{H}_{2} \mathrm{C}=\mathrm{CHCH}_{2} \mathrm{O}\right), 54.5(\mathrm{C}-2), 25.5\left[\left(\mathrm{CH}_{3}\right)_{3} \mathrm{CSi}\right], 23.4$ $\left(\mathrm{CH}_{3} \mathrm{CH}_{2} \mathrm{~S}\right), 20.7(2 \mathrm{C})$ and $20.5\left(3 \mathrm{COCH}_{3}\right), 17.9\left[\left(\mathrm{CH}_{3}\right)_{3} \mathrm{CSi}\right], 14.6\left(\mathrm{CH}_{3} \mathrm{CH}_{2} \mathrm{~S}\right)$, $-5.9\left[\left(\mathrm{CH}_{3}\right)_{2} \mathrm{Si}\right]$. Anal. Calcd for $\mathrm{C}_{63} \mathrm{H}_{77} \mathrm{NO}_{19} \mathrm{SiS}: \mathrm{C}, 62.4 ; \mathrm{H}, 6.4$. Found: $\mathrm{C}, 62.0 ; \mathrm{H}$, 6.0 .

Methyl 3,6-di-O-benzyl-2-deoxy-2-phthalimido- $\beta$-D-glucopyranoside (11).-A solution of cthyl 3,6-di- $O$-benzyl-2-deoxy-2-phthalimido-1-thio- $\beta$-D-glucopyranoside [10] (10; $558 \mathrm{mg}, 1.05 \mathrm{mmol})$ in toluene $(10 \mathrm{~mL})$ and $\mathrm{MeOH}(800 \mu \mathrm{L}, 20 \mathrm{mmol})$ containing powdered $4 \mathrm{~A}$ molecular sieves $\left(2 \mathrm{~g}\right.$ ) was stirred for $30 \mathrm{~min}$ under $\mathrm{N}_{2}$. Methyl triflate ( 553 $\mu \mathrm{L}, 5.04 \mathrm{mmol}$ ) was added at room temperature and the mixture was stirred for $16 \mathrm{~h}$, when TLC (7:3 hexane-EtOAc) showed the conversion of $10\left(R_{f} 0.28\right)$ into $11\left(R_{f} 0.21\right)$ to be 
complete. Triethylamine ( $5 \mathrm{~mL}$ ) was added, and the mixture was diluted with $\mathrm{CH}_{2} \mathrm{Cl}_{2}$ ( 300 $\mathrm{mL})$, filtered through Celite, washed with water, dried $\left(\mathrm{MgSO}_{4}\right)$, filtered, and concentrated. Column chromatography ( $7: 3$ hexane-EtOAc) of the residue gave 11, isolated as a syrup $(336 \mathrm{mg}, 66 \%) ;[\alpha]_{\mathrm{D}}+40^{\circ}(c 1)$; lit $[18][\alpha]_{\mathrm{D}}+39.4^{\circ}(c 1.9) ; R_{f} 0.21$. NMR data $\left(\mathrm{CDCl}_{3}\right):{ }^{1} \mathrm{H}, \delta 7.71-6.91(\mathrm{~m}, 14 \mathrm{H}, 2 \mathrm{Ph}$ and $\mathrm{Phth}), 5.072\left(\mathrm{~d}, 1 \mathrm{H}, J_{1,2} 8.2 \mathrm{~Hz}, \mathrm{H}-1\right)$, 4.752 and $4.526\left(2 \mathrm{~d}\right.$, each $\left.1 \mathrm{H}, \mathrm{PhCH}_{2} \mathrm{O}\right), 4.655$ and $4.589\left(2 \mathrm{~d}\right.$, each $\left.1 \mathrm{H}, \mathrm{PhCH}_{2} \mathrm{O}\right)$, $3.374\left(\mathrm{~s}, 3 \mathrm{H}, \mathrm{CH}_{3} \mathrm{O}\right.$ ), 3.018 (bs, $\left.1 \mathrm{H}, \mathrm{HO}-4\right) ;{ }^{13} \mathrm{C}, \delta 167.9$ and 167.5 (CO Phth), 137.9, 137.5, 133.5, 131.4, 128.2-127.1, and $123.0\left(\mathrm{C}_{6} \mathrm{H}_{5} \mathrm{CH}_{2} \mathrm{O}\right.$ and Phth $), 98.9(\mathrm{C}-1), 78.5$ and 73.8 ( $2 \mathrm{C}$ ) $(\mathrm{C}-3,4,5), 74.0,73.4$, and $70.2\left(\mathrm{C}-6\right.$ and $\left.2 \mathrm{PhCH}_{2} \mathrm{O}\right), 56.3$ and $55.0(\mathrm{C}-2$ and $\mathrm{CH}_{3} \mathrm{O}$ ). Anal. Calcd for $\mathrm{C}_{29} \mathrm{H}_{29} \mathrm{NO}_{7} \cdot 0.5 \mathrm{H}_{2} \mathrm{O}: \mathrm{C}, 67.96 ; \mathrm{H}, 5.90$. Found: $\mathrm{C}, 68.00 ; \mathrm{H}, 5.63$.

Methyl 4-O-\{4-O-[3-O-allyl-4-O-benzoyl-6-O-tert-butyldimethylsilyl-2-0-(2,3,4-triO-acetyl- $\beta$-D-xylopyranosyl)- $\beta$-D-mannopyranosyl $7-3,6$-di-O-benzyl-2-deoxy-2-phthalimido- $\beta$-D-glucopyranosyl $\}-3,6$-di-O-benzyl-2-deoxy-2-phthalimido- $\beta$-D-glucopyranoside (12).-A solution of $9(510 \mathrm{mg}, 0.42 \mathrm{mmol})$ and $11(335 \mathrm{mg}, 0.67 \mathrm{mmol})$ in $1: 1$ 1,2-dichloroethane-toluene $(10 \mathrm{~mL})$ containing powdered $4 \mathrm{~A}$ molecular sieves $(2.5 \mathrm{~g})$ was stirred for $30 \mathrm{~min}$ under $\mathrm{N}_{2}$. Then a solution of $N$-iodosuccinimide ( $115 \mathrm{mg}, 0.51$ $\mathrm{mmol})$ and trifluoromethanesulfonic acid $(6 \mathrm{~mL}, 68 \mathrm{mmol})$ in $1: 11,2$-dichloroethaneether $(5 \mathrm{~mL})$ was added at room temperature. After $15 \mathrm{~min}$, TLC (1:1 hexane-EtOAc) showed the disappearance of $9\left(R_{f} 0.49\right)$, and a new compound $12\left(R_{f} 0.36\right)$. The mixture was diluted with $\mathrm{CH}_{2} \mathrm{Cl}_{2}$ ( $500 \mathrm{~mL}$ ), filtered through Celite, washed with aq $5 \% \mathrm{Na}_{2} \mathrm{~S}_{2} \mathrm{O}_{3}$ $(2 \times 50 \mathrm{~mL})$, aq $5 \% \mathrm{NaHCO}_{3}(2 \times 50 \mathrm{~mL})$, and water, dried $\left(\mathrm{MgSO}_{4}\right)$, filtered, and concentrated. Column chromatography $(3: 2$ hexane-EtOAc) of the residue gave 12, isolated as a white foam $(364 \mathrm{mg}, 52 \%) ;[\alpha]_{\mathrm{D}}-25^{\circ}(c 1) ; R_{f} 0.16$. NMR data $\left(\mathrm{CDCl}_{3}\right):{ }^{1} \mathrm{H}$, $\delta 8.02-6.73(\mathrm{~m}, 33 \mathrm{H}, 5 \mathrm{Ph}$ and $2 \mathrm{Phth}), 5.688\left(\mathrm{~m}, 1 \mathrm{H}, \mathrm{H}_{2} \mathrm{C}=\mathrm{CHCH}_{2} \mathrm{O}\right), 5.332(\mathrm{t}, 1 \mathrm{H}$, $\left.J_{4^{\prime \prime}, 3^{\prime \prime}}=J_{4^{\prime \prime}, 5^{\prime \prime}}=9.8 \mathrm{~Hz}, \mathrm{H}-4^{\prime \prime}\right), 5.290\left(\mathrm{~d}, 1 \mathrm{H}, J_{1,2} 8.3 \mathrm{~Hz}, \mathrm{H}-1\right), 5.175\left(\mathrm{~d}, 1 \mathrm{H}, J_{1^{\prime \prime \prime}, 2^{\prime \prime \prime}} 1.8 \mathrm{~Hz}\right.$, H-1"'), 5.128 and $5.028\left(2 \mathrm{~m}, 2 \mathrm{H}, \mathrm{H}_{2} \mathrm{C}=\mathrm{CHCH}_{2} \mathrm{O}\right), 5.007$ (d, $\left.1 \mathrm{H}, \mathrm{PhCH} H \mathrm{O}\right), 4.866(\mathrm{~d}$, $\left.1 \mathrm{H}, J_{1^{\prime}, 2^{\prime}} 7.9 \mathrm{~Hz}, \mathrm{H}-1^{\prime}\right), 4.864$ (d, $\left.1 \mathrm{H}, \mathrm{PhCH} H \mathrm{O}\right), 4.757\left(\mathrm{~m}, 1 \mathrm{H}, \mathrm{H}-4^{\prime \prime \prime}\right), 4.666$ (s, $1 \mathrm{H}$, $\left.\mathrm{H}-1^{\prime \prime}\right), 3.820\left(\mathrm{~m}, 1 \mathrm{H}, \mathrm{H}_{2} \mathrm{C}=\mathrm{CHCH} H \mathrm{O}\right), 3.274\left(\mathrm{~s}, 3 \mathrm{H}, \mathrm{CH}_{3} \mathrm{O}\right), 2.132,2.124$, and 2.084 ( $3 \mathrm{~s}$, each $3 \mathrm{H}, 3 \mathrm{Ac}$ ), 0.668 [s, $9 \mathrm{H},\left(\mathrm{CH}_{3}\right)_{3} \mathrm{CSi}$ ], -0.183 and -0.248 [ $2 \mathrm{~s}$, each $3 \mathrm{H}$, $\left.\left(\mathrm{CH}_{3}\right)_{2} \mathrm{Si}\right] ;{ }^{13} \mathrm{C}, \delta 169.5,169.4$, and $168.4\left(3 \mathrm{COCH}_{3}\right), 168.0$ and 167.3 ( $\mathrm{CO}$ Phth ), 164.9 (COPh) , 138.9, 138.3, 138.1, 137.8, 134.1, 133.3, 132.6, 131.4, 131.1, 129.9, 129.3, 128.2126.3, and $122.8 \quad\left(C_{6} \mathrm{H}_{5} \mathrm{CH}_{2} \mathrm{O}, \quad C_{6} \mathrm{H}_{5} \mathrm{CO}, \quad \mathrm{H}_{2} \mathrm{C}=\mathrm{CHCH}_{2} \mathrm{O}\right.$, and Phth $), 116.6$ $\left(\mathrm{H}_{2} \mathrm{C}=\mathrm{CHCH}_{2} \mathrm{O}\right), 100.9,98.8,96.5$, and $96.7\left(\mathrm{C}-1,1^{\prime}, 1^{\prime \prime}, 1^{\prime \prime \prime}\right), 79.8,78.3,77.4,75.3,75.1$,

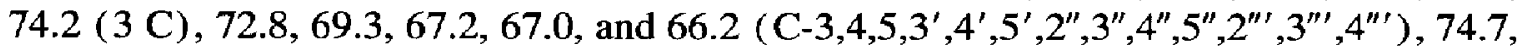
$74.0,73.0,72.4,70.4,68.0,67.8,63.5$, and $58.3\left(\mathrm{C}-6,6^{\prime}, 6^{\prime \prime}, 5^{\prime \prime \prime}, 4 \mathrm{PhCH}_{2} \mathrm{O}\right.$, and $\left.\mathrm{H}_{2} \mathrm{C}=\mathrm{CHCH}_{2} \mathrm{O}\right), 56.3,56.1$, and $55.3\left(\mathrm{C}-2,2^{\prime}\right.$ and $\left.\mathrm{CH}_{3} \mathrm{O}\right), 25.4\left[\left(\mathrm{CH}_{3}\right)_{3} \mathrm{CSi}\right], 20.7,20.6$, and $20.5\left(3 \mathrm{COCH}_{3}\right), 17.8\left[\left(\mathrm{CH}_{3}\right)_{3} \mathrm{CSi}\right],-6.0\left[\left(\mathrm{CH}_{3}\right)_{2} \mathrm{Si}\right]$. Anal. Calcd for $\mathrm{C}_{90} \mathrm{H}_{100} \mathrm{~N}_{2} \mathrm{O}_{26} \mathrm{Si}: \mathrm{C}, 65.36 ; \mathrm{H}, 6.09$. Found: $\mathrm{C}, 65.07 ; \mathrm{H}, 6.29$.

Methyl 4-O- $\{4-\mathrm{O}-[3-\mathrm{O}-a l l y l-4-\mathrm{O}-$ benzoyl-2-O- $(2,3,4-t r i-\mathrm{O}-a c e t y l-\beta-\mathrm{D}-x y l o p y r a n o-$ syl)- $\beta$-D-mannopyranosyl]-3, 6-di-O-benzyl-2-deoxy-2-phthalimido- $\beta$-D-glucopyranosyl\}-3,6-di-O-benzyl-2-deoxy-2-phthalimido- $\beta$-D-glucopyranoside $(13) .-\Lambda$ solution of $12(266 \mathrm{mg}, 0.16 \mathrm{mmol})$ and $p$-toluenesulfonic acid monohydrate $(150 \mathrm{mg}, 0.79 \mathrm{mmol})$ in 9:1 MeCN-water $(15 \mathrm{~mL})$ was stirred for $30 \mathrm{~min}$ at room temperature. TLC $\left(9: 1 \mathrm{CH}_{2} \mathrm{Cl}_{2}-\right.$ acetone) then showed a complete desilylation, and the solution was diluted with $\mathrm{CH}_{2} \mathrm{Cl}_{2}$ $(250 \mathrm{~mL})$, washed with aq $5 \% \mathrm{NaHCO}_{3}(25 \mathrm{~mL})$ and water, dried $\left(\mathrm{MgSO}_{4}\right)$, filtered, and 
concentrated. Column chromatography $\left(9: 1 \mathrm{CH}_{2} \mathrm{Cl}_{2}\right.$-acetone) of the residue gave 13, isolated as a white foam $(241 \mathrm{mg}, 97 \%) ;[\alpha]_{\mathrm{D}}-29^{\circ}(c 1) ; R_{f} 0.30$. NMR data $\left(\mathrm{CDCl}_{3}\right)$ : ${ }^{1} \mathrm{H}, \delta 7.98-6.74(\mathrm{~m}, 33 \mathrm{H}, 5 \mathrm{Ph}$ and $2 \mathrm{Phth}), 5.726\left(\mathrm{~m}, 1 \mathrm{H}, \mathrm{H}_{2} \mathrm{C}=\mathrm{CHCH}_{2} \mathrm{O}\right), 5.330(\mathrm{~d}, 1$ $\left.\mathrm{H}, J_{1,2} 8.4 \mathrm{~Hz}, \mathrm{H}-1\right), 5.253\left(\mathrm{t}, 1 \mathrm{H}, J_{4^{\prime \prime}, 3^{\prime \prime}}=J_{4^{\prime \prime}, 5^{\prime \prime}}=9.6 \mathrm{~Hz}, \mathrm{H}-4^{\prime \prime}\right), 5.154$ and $5.059(2 \mathrm{~m}, 2$ $\mathrm{H}, \mathrm{H}_{2} \mathrm{C}=\mathrm{CHCH}_{2} \mathrm{O}$ ), 5.125 (d, $\left.1 \mathrm{H}, J_{1^{\prime \prime}, 2^{\prime \prime \prime}} 1.5 \mathrm{~Hz}, \mathrm{H}-1^{\prime \prime \prime}\right), 5.038$ (d, $1 \mathrm{H}, \mathrm{PhCH} H \mathrm{O}$ ), 4.889 (d, $1 \mathrm{H}, \mathrm{PhCH} H \mathrm{O}), 4.881$ (d, $1 \mathrm{H}, J_{1^{\prime}, 2^{\prime}} 7.8 \mathrm{~Hz}, \mathrm{H}-1^{\prime}$ ) , 4.764 (m, $1 \mathrm{H}, \mathrm{H}-4^{\prime \prime \prime}$ ) , 4.633 (s, 1 $\left.\mathrm{H}, \mathrm{H}-1^{\prime \prime}\right), 3.846\left(\mathrm{~m}, 1 \mathrm{H}, \mathrm{H}_{2} \mathrm{C}=\mathrm{CHCH} H \mathrm{O}\right.$ ), 3.282 ( $\left.\mathrm{s}, 3 \mathrm{H}, \mathrm{CH}_{3} \mathrm{O}\right), 2.154,2.133$, and 2.112 $(3 \mathrm{~s}$, each $3 \mathrm{H}, 3 \mathrm{Ac}) ;{ }^{13} \mathrm{C}, \delta 169.7,169.6$, and $168.6\left(3 \mathrm{COCH}_{3}\right), 168.3$ and $167.5(\mathrm{CO}$ Phth), 165.4 ( $C O \mathrm{OPh}), 138.7,138.5,138.3,137.8,134.1,133.7,133.4,133.1,131.6,131.2$, 129.5, 128.5-126.5, 123.4, and 123.0 $\left(\mathrm{C}_{6} \mathrm{H}_{5} \mathrm{CH}_{2} \mathrm{O}, \mathrm{C}_{6} \mathrm{H}_{5} \mathrm{CO}, \mathrm{H}_{2} \mathrm{C}=\mathrm{CHCH}_{2} \mathrm{O}\right.$, and Phth), $116.9\left(\mathrm{H}_{2} \mathrm{C}=\mathrm{CHCH}_{2} \mathrm{O}\right), 100.7,98.9,97.7$, and $96.8\left(\mathrm{C}-1,1^{\prime}, 1^{\prime \prime}, 1^{\prime \prime \prime}\right), 80.1,78.2$, $77.0,76.6,75.6,74.5,74.3,74.2,73.3,68.1,67.1(2 \mathrm{C})$, and 66.3 (C$\left.3,4,5,3^{\prime}, 4^{\prime}, 5^{\prime}, 2^{\prime \prime}, 3^{\prime \prime}, 4^{\prime \prime}, 5^{\prime \prime}, 2^{\prime \prime \prime}, 3^{\prime \prime \prime}, 4^{\prime \prime \prime}\right), 74.6,74.3,73.2,72.5$ (2 C), 70.5, 68.0, 61.7, and 58.5 ( C-6, 6', 6", $5^{\prime \prime \prime}, 4 \mathrm{PhCH}_{2} \mathrm{O}$, and $\left.\mathrm{H}_{2} \mathrm{C}=\mathrm{CHCH}_{2} \mathrm{O}\right), 56.4,56.3$, and $55.4\left(\mathrm{C}-2,2^{\prime}\right.$ and $\mathrm{CH}_{3} \mathrm{O}$ ), $20.8(2 \mathrm{C})$ and $20.6\left(3 \mathrm{COCH}_{3}\right)$. Anal. Calcd for $\mathrm{C}_{84} \mathrm{H}_{86} \mathrm{~N}_{2} \mathrm{O}_{26} \cdot 0.5 \mathrm{H}_{2} \mathrm{O}: \mathrm{C}, 65.15 ; \mathrm{H}, 5.66$. Found: C, 65.05; H, 5.66.

Methyl

4-O- $\{4-\mathrm{O}-13-\mathrm{O}$-allyl-4-O-benzoyl-6-O-(2,3,4,6-tetra-O-acetyl- $\alpha$-D-mannopyranosyl)-2-O-(2, 3, 4-tri-O-acetyl- $\beta$-D-xylopyranosyl)- $\beta$-D-mannopyranosyl]-3, $6-$ di-O-benzyl-2-deoxy-2-phthalimido- $\beta$-D-glucopyranosyl\}-3, 6 -di-O-benzyl-2-deoxy-2phthalimido- $\beta$-D-glucopyranoside (14).-A solution of 13 ( $99 \mathrm{mg}, 64 \mathrm{mmol}$ ) and 2,3,4,6tetra- $O$-acetyl- $\alpha$-D-mannopyranosyl trichloroacetimidate [9] $(7 ; 110 \mathrm{mg}, 0.22 \mathrm{mmol})$ in $\mathrm{CH}_{2} \mathrm{Cl}_{2}(3 \mathrm{~mL})$ containing powdered $4 \mathrm{~A}$ molecular sieves $\left(250 \mathrm{mg}\right.$ ) was stirred under $\mathrm{N}_{2}$ for $30 \mathrm{~min}$. Trimethylsilyl triflate $(10 \mathrm{~mL}, 55 \mu \mathrm{mol})$ was added at $-20^{\circ} \mathrm{C}$ and the mixture was stirred for $30 \mathrm{~min}$ at $0^{\circ} \mathrm{C}$. TLC $\left(95: 5 \mathrm{CH}_{2} \mathrm{Cl}_{2}\right.$-acetone $)$ then showed the disappearance of $13\left(R_{f} 0.37\right)$ and a new product $14\left(R_{f} 0.31\right)$. Pyridine $(1 \mathrm{~mL})$ was added, and the mixture was diluted with $\mathrm{CH}_{2} \mathrm{Cl}_{2}(100 \mathrm{~mL})$, filtered through Celite, washed with water, dried $\left(\mathrm{MgSO}_{4}\right)$, filtered, and concentrated. Column chromatography (37:3 $\mathrm{CH}_{2} \mathrm{Cl}_{2}-\mathbf{a c e}$ tone) of the residue gave 14, isolated as a white foam ( $86 \mathrm{mg}, 71 \%) ;[\alpha]_{\mathrm{D}}-11^{\circ}(c 1)$; $R_{f}$ 0.31. NMR data $\left(\mathrm{CDCl}_{3}\right):{ }^{1} \mathrm{H}, \delta 8.01-6.76(\mathrm{~m}, 33 \mathrm{H}, 5 \mathrm{Ph}$ and $2 \mathrm{Phth}), 5.702(\mathrm{~m}, 1 \mathrm{H}$, $\mathrm{H}_{2} \mathrm{C}=\mathrm{CHCH}_{2} \mathrm{O}$ ), 5.324 ( t, $1 \mathrm{II}, J_{4^{\prime \prime}, 3^{\prime \prime}}=J_{4^{\prime \prime}, 5^{\prime \prime}}=9.8 \mathrm{IIz}, \mathrm{II}-4^{\prime \prime}$ ), 5.304 (d, $1 \mathrm{II}, J_{1,2} 8.5 \mathrm{~Hz}, \mathrm{H}-$ 1), $5.058\left(\mathrm{~m}, 1 \mathrm{H}, \mathrm{HHC}=\mathrm{CHCH}_{2} \mathrm{O}\right), 4.869\left(\mathrm{~d}, 1 \mathrm{H}, J_{1^{m m}, 2^{m \prime \prime}} 1.6 \mathrm{~Hz}, \mathrm{H}-1^{m \prime \prime \prime}\right), 4.766(\mathrm{~m}, 1 \mathrm{H}$,

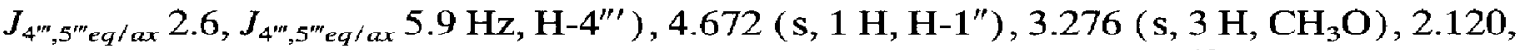
$2.117,2.063,1.989,1.957,1.947$, and 1.857 ( $7 \mathrm{~s}$, each $3 \mathrm{H}, 7 \mathrm{Ac}) ;{ }^{13} \mathrm{C}, \delta 170.4-167.3$ $\left(\mathrm{COCH}_{3}\right.$ and $\left.\mathrm{CO} \mathrm{Phth}\right), 165.3(\mathrm{COPh}), 138.7,138.5,138.3,138.0,134.1,133.4,133.0$, $131.6,131.4,129.5,129.4,128.5-126.7,123.3$, and $123.0\left(C_{6} \mathrm{H}_{5} \mathrm{CH}_{2} \mathrm{O}, C_{6} \mathrm{H}_{5} \mathrm{CO}\right.$, $\mathrm{H}_{2} \mathrm{C}=\mathrm{CHCH}_{2} \mathrm{O}$, and $\left.\mathrm{Phth}\right), 116.9\left(\mathrm{H}_{2} \mathrm{C}=\mathrm{CHCH}_{2} \mathrm{O}\right), 101.0,98.9,97.8$, and 97.1 (2 C) (C-1,1', $\left.1^{\prime \prime}, 1^{\prime \prime \prime}, 1^{\prime \prime \prime \prime}\right), 80.3,78.0,77.0,76.8,75.7,74.4,74.3,72.9,72.5,69.6,69.2,68.6$, $68.4,67.5,67.2,66.5$, and $65.8\left(\mathrm{C}-3,4,5,3^{\prime}, 4^{\prime}, 5^{\prime}, 2^{\prime \prime}, 3^{\prime \prime}, 4^{\prime \prime}, 5^{\prime \prime}, 2^{\prime \prime \prime}, 3^{\prime \prime \prime}, 4^{\prime \prime \prime}, 2^{\prime \prime \prime \prime}, 3^{\prime \prime \prime}, 4^{\prime \prime \prime}, 5^{\prime \prime \prime}\right), 74.8$, 74.3, 73.2 (2 C) , 72.5, 70.8, 68.0 (2 C), 62.2, and 58.6 (C-6, $6^{\prime}, 6^{\prime \prime}, 5^{\prime \prime \prime}, 6^{\prime \prime \prime \prime}, 4 \mathrm{PhCH}_{2} \mathrm{O}$, and $\left.\mathrm{H}_{2} \mathrm{C}=\mathrm{CHCH}_{2} \mathrm{O}\right), 56.3(2 \mathrm{C})$ and $55.4\left(\mathrm{C}-2,2^{\prime}\right.$ and $\left.\mathrm{CH}_{3} \mathrm{O}\right), 20.9,20.7,20.6,20.5(2 \mathrm{C})$, and $20.4(2 \mathrm{C})\left(7 \mathrm{COCH}_{3}\right)$.

Methyl 4-O-\{4-O-[4-O-benzoyl-6-O-(2,3,4, 6-tetra-O-acetyl- $\alpha$-D-mannopyranosyl)$2-\mathrm{O}-(2,3,4-$ tri-O-acetyl- $\beta$-D-xylopyranosyl)- $\beta$-D-mannopyranosyl]-3, 6 -di-O-benzyl-2deoxy-2-phthalimido- $\beta$-D-glucopyranosyl\}-3,6-di-O-benzyl-2-deoxy-2-phthalimido- $\beta$-Dglucopyranoside (15). - A solution of 14 ( $83 \mathrm{mg}, 44 \mathrm{mmol}$ ), tris (triphenylphosphine)- 
rhodium(I) chloride (23 $\mathrm{mg}, 25 \mu \mathrm{mol}$ ), and 1,4-diazabicyclo[2.2.2] octane ( $9 \mathrm{mg}, 80$ $\mu \mathrm{mol})$ in $7: 3: 1 \mathrm{EtOH}-$ toluene-water $(6 \mathrm{~mL})$ was boiled under reflux for $2.5 \mathrm{~h}$, then cooled, and concentrated. A solution of the residue in $9: 1$ acetone- $\mathrm{M} \mathrm{aq} \mathrm{HCl}(9 \mathrm{~mL})$ was boiled under reflux for $30 \mathrm{~min}$, when TLC $\left(7: 1 \mathrm{CH}_{2} \mathrm{Cl}_{2}\right.$-acetone $)$ indicated a complete conversion of 14 into $15\left(R_{f} 0.65\right)$. Then the solution was concentrated, and a solution of the residue in $\mathrm{CH}_{2} \mathrm{Cl}_{2}(100 \mathrm{~mL})$ was washed with aq $5 \% \mathrm{NaHCO}_{3}(10 \mathrm{~mL})$ and water, dried $\left(\mathrm{MgSO}_{4}\right)$, filtered, and concentrated. Column chromatography $\left(7: 1 \mathrm{CH}_{2} \mathrm{Cl}_{2}\right.$-acetone) of the residue gave 15, isolated as a syrup $(71 \mathrm{mg}, 85 \%) ;[\alpha]_{\mathrm{D}}+5^{\circ}(c 1) ; R_{f} 0.32$. NMR data $\left(\mathrm{CDCl}_{3}\right)$ : ${ }^{1} \mathrm{H}, \delta 8.02-6.72(\mathrm{~m}, 33 \mathrm{H}, 5 \mathrm{Ph}$ and $2 \mathrm{Phth}), 4.631\left(\mathrm{~s}, 1 \mathrm{H}, \mathrm{H}-1^{\prime \prime}\right), 3.269\left(\mathrm{~s}, 3 \mathrm{H}, \mathrm{CH}_{3} \mathrm{O}\right)$, $2.106,2.102,2.028,2.000,1.936,1.910$, and $1.893(7 \mathrm{~s}$, each $3 \mathrm{H}, 7 \mathrm{Ac}) ;{ }^{13} \mathrm{C}, \delta 170.4$, $169.7,169.6$ (2 C) $, 169.4,169.3,168.8\left(7 \mathrm{COCH}_{3}\right), 168.1-167.3$ ( $\mathrm{CO}$ Phth), 165.7 (COPh), 138.5 (2 C), 138.2, 137.7, 133.4, 131.6, 131.2, 129.6, 129.3, 128.5-126.7, and $123.0\left(C_{6} \mathrm{H}_{5} \mathrm{CH}_{2} \mathrm{O}, C_{6} \mathrm{H}_{5} \mathrm{CO}\right.$, and $\left.\mathrm{Phth}\right), 101.3,99.3,98.8$, and 97.0 (2 C) (C$\left.1,1^{\prime}, 1^{\prime \prime}, 1^{\prime \prime \prime}, 1^{\prime \prime \prime}\right), 56.3(2 \mathrm{C})$ and $55.4\left(\mathrm{C}-2,2^{\prime}\right.$ and $\left.\mathrm{CH}_{3} \mathrm{O}\right), 20.7-20.4\left(\mathrm{COCH}_{3}\right)$. Anal. Calcd for $\mathrm{C}_{95} \mathrm{H}_{100} \mathrm{~N}_{2} \mathrm{O}_{35} \cdot \mathrm{H}_{2} \mathrm{O}: \mathrm{C}, 61.75 ; \mathrm{H}, 5.56$. Found: $\mathrm{C}, 61.54 ; \mathrm{H}, 5.64$.

Methyl 4-O-\{4-O-[4-O-benzoyl-3, 6-di-O-(2, 3, 4, 6-tetra-O-acetyl- $\alpha$-D-mannopyranosyl)-2-O-(2, 3, 4-tri-O-acetyl- $\beta$-D-xylopyranosyl)- $\beta$-D-mannopyranosyl $/$-3, 6-di-O-benzyl-2-deoxy-2-phthalimido- $\beta$-D-glucopyranosyl $\}-3,6$-di-O-benzyl-2-deoxy-2-phthalimido$\beta$-D-glucopyranoside (16). - A solution of 15 (39 $\mathrm{mg}, 21 \mu \mathrm{mol}$ ) and 2,3,4,6-tetra-Oacetyl- $\alpha$-D-mannopyranosyl trichloroacetimidate [9] ( 7; $105 \mathrm{mg}, 0.21 \mathrm{mmol}$ ) in $\mathrm{CH}_{2} \mathrm{Cl}_{2}$ ( $2 \mathrm{~mL}$ ) containing powdered $4 \mathrm{~A}$ molecular sieves $\left(100 \mathrm{mg}\right.$ ) was stirred under $\mathrm{N}_{2}$ for 30 min. Trimethylsilyl triflate ( $3 \mu \mathrm{L}, 17 \mu \mathrm{mol}$ ) was added and the mixture was stirred for $2 \mathrm{~h}$ at room temperature. TLC (95:5 $\mathrm{CH}_{2} \mathrm{Cl}_{2}$-acetone) then showed the disappearance of $\mathbf{1 5}$ $\left(R_{f} 0.73\right)$, and a new product $16\left(R_{f} 0.64\right)$. Pyridine ( $\left.1 \mathrm{~mL}\right)$ was added, and the mixture was diluted with $\mathrm{CH}_{2} \mathrm{Cl}_{2}(100 \mathrm{~mL})$, filtered through Celite, washed with water, dried $\left(\mathrm{MgSO}_{4}\right)$, filtered, and concentrated. Column chromatography $(3: 7$ hexane-EtOAc) of the residue gave 16, isolated as a syrup $(27 \mathrm{mg}, 59 \%) ;[\alpha]_{\mathrm{D}}-2^{\circ}(c 1) ; R_{f} 0.33$. NMR data $\left(\mathrm{CDCl}_{3}\right):{ }^{1} \mathrm{H}, \delta 7.97-6.74(\mathrm{~m}, 33 \mathrm{H}, 5 \mathrm{Ph}$ and $2 \mathrm{Phth}), 5.409\left(\mathrm{t}, 1 \mathrm{H}, J_{4^{\prime \prime}, 3^{\prime \prime}}=J_{4^{\prime \prime}, 5^{\prime \prime}}=9.8 \mathrm{~Hz}\right.$, $\left.\mathrm{H}-4^{\prime \prime}\right), 5.163$ (d, $\left.1 \mathrm{H}, J_{1^{\prime \prime \prime \prime}, 2^{\prime \prime \prime \prime}} 1.4 \mathrm{~Hz}, \mathrm{H}-1^{\prime \prime \prime \prime \prime}\right), 4.983$ (d, $\left.1 \mathrm{H}, J_{1^{\prime \prime \prime}, 2^{m \prime \prime}} 1.8 \mathrm{~Hz}, \mathrm{H}-1^{\prime \prime \prime \prime}\right), 3.271$ $\left(\mathrm{s}, 3 \mathrm{H}, \mathrm{CH}_{3} \mathrm{O}\right), 2.119,2.106,2.081,2.056,1.999,1.995,1.951,1.881,1.857$, and 1.824 $(10 \mathrm{~s}, 3,3,3,3,3,3,6,3,3,3 \mathrm{H}, 11 \mathrm{Ac}) ;{ }^{13} \mathrm{C}, \delta$ 170.5-167.3 $\left(\mathrm{COCH}_{3}\right.$ and $\mathrm{CO}$ Phth $), 165.0$ (COPh), 138.6, 138.5, 138.3, 138.0, 133.4, 133.2, 131.6, 131.4, 129.6, 128.6-126.7, and $123.0\left(C_{6} \mathrm{H}_{5} \mathrm{CH}_{2} \mathrm{O}, C_{6} \mathrm{H}_{5} \mathrm{CO}\right.$, and $\mathrm{Phth}$ ), 101.0, 99.3, 98.9, 98.2, and 97.0 (2 C) (C$\left.1,1^{\prime}, 1^{\prime \prime}, 1^{\prime \prime \prime}, 1^{\prime \prime \prime}, 1^{\prime \prime \prime \prime \prime}\right), 56.3,56.2$, and $55.4\left(\mathrm{C}-2,2^{\prime}\right.$ and $\left.\mathrm{CH}_{3} \mathrm{O}\right), 20.8-20.2\left(\mathrm{COCH}_{3}\right)$. Anal. Calcd for $\mathrm{C}_{109} \mathrm{H}_{118} \mathrm{~N}_{2} \mathrm{O}_{44}: \mathrm{C}, 60.61 ; \mathrm{H}, 5.51$. Found: $\mathrm{C}, 60.37 ; \mathrm{H}, 5.52$.

Methyl 2-acetamido-4-O-[2-acetamido-2-deoxy-4-O-(3,6-di-O- $\alpha$-D-mannopyranosyl$2-\mathrm{O}-\beta$-D-xylopyranosyl- $\beta$-D-mannopyranosyl)- $\beta$-D-glucopyranosyl]-2-deoxy- $\beta$-D-glucopyranoside (4).-A solution of $16(26 \mathrm{mg}, 12 \mu \mathrm{mol})$ in $\mathrm{MeNH}_{2}-\mathrm{EtOH}(33 \%, 10 \mathrm{~mL})$ was stirred for $32 \mathrm{~h}$, then concentrated, and treated with $\mathrm{Ac}_{2} \mathrm{O}(100 \mathrm{~mL}, 1 \mu \mathrm{mol})$ in $\mathrm{MeOH}$ ( $3 \mathrm{~mL}$ ) for $2 \mathrm{~h}$. The solution was concentrated, and toluene, $\mathrm{EtOH}$, and $\mathrm{CH}_{2} \mathrm{Cl}_{2}$ (each $3 \times 5$ $\mathrm{mL}$ ) werc evaporated from the residue. Column chromatography on Scphadex LH-20 (1:1 $\mathrm{MeOH}-\mathrm{CH}_{2} \mathrm{Cl}_{2}$ ) of the residue gave a white solid. To a solution of this product in $\mathrm{MeOH}$ $(4 \mathrm{~mL})$, containing AcOH $(50 \mu \mathrm{L})$, was added $10 \% \mathrm{Pd}-\mathrm{C}(26 \mathrm{mg})$, and hydrogenolysis was performed at atmospheric pressure for $32 \mathrm{~h}$. Then the mixture was filtered through Celite and concentrated, and toluene, $\mathrm{EtOH}$, and $\mathrm{CH}_{2} \mathrm{Cl}_{2}($ each $3 \times 10 \mathrm{~mL})$ were evaporated 
from the residue. Because ${ }^{1} \mathrm{H}$ NMR analysis showed that the dephthaloylation was incomplete, a solution of the residue and hydrazine acetate $(60 \mathrm{mg}, 0.65 \mathrm{mmol})$ in EtOH ( $5 \mathrm{~mL})$ was boiled under reflux for $16 \mathrm{~h}$, then concentrated and treated with $\mathrm{Ac}_{2} \mathrm{O}$ ( $300 \mu \mathrm{L}, 3.1$ $\mathrm{mmol}$ ) in $\mathrm{MeOH}(3 \mathrm{~mL})$ for $16 \mathrm{~h}$. The solution was concentrated, and toluene, EtOH, and $\mathrm{CH}_{2} \mathrm{Cl}_{2}$ (each $3 \times 10 \mathrm{~mL}$ ) were evaporated from the residue, which was fractionated on Sephadex LH-20 (1:1 MeOH-CH $\left.\mathrm{Cl}_{2}\right)$ to yield impure 4. This product was then subjected to purification by RP-HPLC on a Cp tm Spher C18 column $(250 \times 4.6 \mathrm{~mm}$, Chrompack) by elution with 95:5 water-MeOH for $5 \mathrm{~min}$, followed by a linear gradient of 95:5 $\rightarrow 1: 1$ water-MeOH for $35 \mathrm{~min}$, and detection at $205 \mathrm{~nm}$. The fraction eluting at $5.9 \mathrm{~min}$ was collected and concentrated to yield 4 , isolated as a white amorphous solid ( $9.8 \mathrm{mg}, 77 \%)$; $[\alpha]_{\mathrm{D}}-8^{\circ}\left(c 0.1, \mathrm{H}_{2} \mathrm{O}\right)$. For ${ }^{1} \mathrm{H}$ NMR data, see Table 1.

Ethyl 3,6-di-O-benzyl-2-deoxy-4-O-[4,6-di-O-acetyl-3-O-allyl-2-O- $(2,3,4-t r i-\mathrm{O}$-acetyl- $\beta$-D-xylopyranosyl)- $\beta$-D-mannopyranosyl]-2-phthalimido-1-thio- $\beta$-D-glucopyranoside (17). - A solution of ethyl 4-O-[3-O-allyl-2- $O$ - (2,3,4-tri- $O$-acetyl- $\beta$-D-xylopyranosyl) - $\beta$-D-mannopyranosyl]-3,6-di- $O$-benzyl-2-deoxy-2-phthalimido-1-thio- $\beta$-D-glucopyranoside [10] $(8 ; 1.34 \mathrm{~g}, 1.35 \mathrm{mmol})$ in $1: 1$ pyridine- $\mathrm{Ac}_{2} \mathrm{O}(20 \mathrm{~mL})$ was kept for $16 \mathrm{~h}$ at room temperature, then concentrated, and toluene, $\mathrm{EtOH}$, and $\mathrm{CH}_{2} \mathrm{Cl}_{2}$ (each $3 \times 20 \mathrm{~mL}$ ) were evaporated from the residue, to give 17 , isolated as a white foam $(1.45 \mathrm{~g}, 99 \%) ;[\alpha]_{\mathrm{D}}$ $-35^{\circ}$ (c 1$) ; R_{f} 0.28\left(1: 1\right.$ hexane-ElOAc). NMR data $\left(\mathrm{CDCl}_{3}\right):{ }^{1} \mathrm{H}, \delta 7.75-6.75(\mathrm{~m}, 14$ $\mathrm{H}, 2 \mathrm{Ph}$ and Phth $), 5.811\left(\mathrm{~m}, 1 \mathrm{H}, \mathrm{H}_{2} \mathrm{C}=\mathrm{CHCH}_{2} \mathrm{O}\right), 5.262\left(\mathrm{~d}, 1 \mathrm{H}, J_{1,2} 10.4 \mathrm{~Hz}, \mathrm{H}-1\right)$, 5.228 and $5.151\left(2 \mathrm{~m}, 2 \mathrm{H}, \mathrm{H}_{2} \mathrm{C}=\mathrm{CHCH}_{2} \mathrm{O}\right), 5.146\left(\mathrm{~d}, 1 \mathrm{H}, J_{1^{\prime \prime}, 2^{\prime \prime}} 2.4 \mathrm{~Hz}, \mathrm{H}-1^{\prime \prime}\right), 5.094$ (t, $\left.1 \mathrm{H}, J_{4^{\prime}, 5^{\prime}} 9.9 \mathrm{~Hz}, \mathrm{H}-4^{\prime}\right), 4.983$ and 4.940 ( $\left.2 \mathrm{bt}, 2 \mathrm{H}, \mathrm{H}-2^{\prime \prime}, 3^{\prime \prime}\right), 4.927$ and 4.369 ( $2 \mathrm{~d}$, each $1 \mathrm{H}, \mathrm{PhCH}_{2} \mathrm{O}$ ), 4.760 (m, $1 \mathrm{H}, J_{4^{\prime \prime}, 5^{\prime \prime a x} / e q} 2.6$ and $\left.2.9 \mathrm{~Hz}, \mathrm{H}-4^{\prime \prime}\right), 4.747$ and 4.534 ( $2 \mathrm{~d}$, each $1 \mathrm{H}, \mathrm{PhCH}_{2} \mathrm{O}$ ) , 4.613 ( $\left.\mathrm{s}, 1 \mathrm{H}, \mathrm{H}-1^{\prime}\right), 4.551$ and 3.524 ( $2 \mathrm{dd}$, each $1 \mathrm{H}, J_{5^{\prime \prime} \alpha x, 5^{\prime \prime} e q}-13.0 \mathrm{~Hz}$, H-5"ax, 5"eq), 4.045 (d, $1 \mathrm{H}, J_{2^{\prime}, 3^{\prime}} 3.0 \mathrm{~Hz}, \mathrm{H}-2^{\prime}$ ) , 3.175 (dd, $1 \mathrm{H}, J_{3^{\prime}, 4^{\prime}} 9.9 \mathrm{~Hz}, \mathrm{H}-3^{\prime}$ ) , 2.637 $\left(\mathrm{m}, 2 \mathrm{H}, \mathrm{CH}_{3} \mathrm{CH}_{2} \mathrm{~S}\right), 2.127,2.122,2.076,2.036$, and $1.934(5 \mathrm{~s}$, each $3 \mathrm{H}, 5 \mathrm{Ac}), 1.175$ $\left(\mathrm{t}, 3 \mathrm{H}, \mathrm{CH}_{3} \mathrm{CH}_{2} \mathrm{~S}\right) ;{ }^{13} \mathrm{C}, \delta 170.5,169.7,169.4(2 \mathrm{C}), 168.6\left(5 \mathrm{COCH}_{3}\right), 138.7,137.8$, 134.0, 133.5, 128.3-126.6, and $123.0\left(\mathrm{C}_{6} \mathrm{H}_{5} \mathrm{CH}_{2} \mathrm{O}, \mathrm{H}_{2} \mathrm{C}=\mathrm{CHCH}_{2} \mathrm{O}\right.$, and Phth), 116.5 $\left(\mathrm{H}_{2} \mathrm{C}=\mathrm{CHCH}_{2} \mathrm{O}\right), 101.5$ and $97.7\left(\mathrm{C}-1^{\prime}, 1^{\prime \prime}\right), 81.0,80.6,78.6,78.4,78.0,72.5,72.1,67.4$, $67.3,67.2$, and $66.5\left(\mathrm{C}-1,3,4,5,2^{\prime}, 3^{\prime}, 4^{\prime}, 5^{\prime}, 2^{\prime \prime}, 3^{\prime \prime}, 4^{\prime \prime}\right), 74.7,73.4,70.2,68.9,62.7$, and 58.6 $\left(\mathrm{C}-6,6^{\prime}, 5^{\prime \prime}, 2 \mathrm{PhCH}_{2} \mathrm{O}\right.$, and $\left.\mathrm{H}_{2} \mathrm{C}=\mathrm{CHCH}_{2} \mathrm{O}\right), 54.6(\mathrm{C}-2), 23.6\left(\mathrm{CH}_{3} \mathrm{CH}_{2} \mathrm{~S}\right), 20.8,20.7$, 20.6, and 20.5 (2 C) ( $\left.5 \mathrm{COCH}_{3}\right), 14.7\left(\mathrm{CH}_{3} \mathrm{CH}_{2} \mathrm{~S}\right)$. Anal. Calcd for $\mathrm{C}_{54} \mathrm{H}_{63} \mathrm{NO}_{20} \mathrm{~S}: \mathrm{C}$, $60.16 ; \mathrm{H}, 5.89$. Found: $\mathrm{C}, 59.97 ; \mathrm{H}, 6.07$.

Methyl 3-O-benzyl-2-deoxy-4-O- $\{3,6$-di-O-benzyl-2-deoxy-4-O-[4,6-di-O-acetyl-3$\mathrm{O}$-allyl-2-O-(2,3,4-tri-O-acetyl- $\beta$-D-xylopyranosyl)- $\beta$-D-mannopyranosyl]-2-phthalimido- $\beta$-D-glucopyranosyl\}-6-O-(4-methoxybenzyl)-2-phthalimido- $\beta$-D-glucopyranoside (19) and 1,5-anhydro-3, 6-di-O-benzyl-2-deoxy-4-0-[4,6-di-O-acetyl-3-O-allyl-2-O$(2,3,4-t r i-\mathrm{O}-$ acetyl- $\beta$-D-xylopyranosyl)- $\beta$-D-mannopyranosyl -2 -phthalimido-D-arabinohex-1-enitol (20). - A solution of $17(637 \mathrm{mg}, 0.59 \mathrm{mmol})$ and methyl 3-O-benzyl-2deoxy-6- $O$-( 4-methoxybenzyl) -2-phthalimido- $\beta$-D-glucopyranoside [18] (18; $470 \mathrm{mg}$, $0.88 \mathrm{mmol})$ in $2: 1$ 1,2-dichloroethane-toluene $(15 \mathrm{~mL})$, containing powdered $4 \mathrm{~A}$ molecular sieves $\left(2 \mathrm{~g}\right.$ ), was stirred for 30 min under $\mathrm{N}_{2}$. A solution of $\mathrm{N}$-iodosuccinimide ( 150 $\mathrm{mg}, 0.67 \mathrm{mmol})$ and trifluoromethanesulfonic acid $(8 \mu \mathrm{L}, 79 \mu \mathrm{mol})$ in 1:1 1,2-dichloroethane-ether $(4 \mathrm{~mL})$ was added at room temperature. After $5 \mathrm{~min}$, TLC ( $1: 1$ hexane-EtOAc) showed the disappearance of $17\left(R_{f} 0.28\right)$, and two new products $20\left(R_{f} 0.20\right)$ and $19\left(R_{f}\right.$ 
0.16). The mixture was diluted with $\mathrm{CH}_{2} \mathrm{Cl}_{2}(500 \mathrm{~mL})$, filtered through Celite, washed with aq $5 \% \mathrm{Na}_{2} \mathrm{~S}_{2} \mathrm{O}_{3}(2 \times 50 \mathrm{~mL})$, aq $5 \% \mathrm{NaHCO}_{3}$, and water, dried $\left(\mathrm{MgSO}_{4}\right)$, filtered, and concentrated. Column chromatography $(1: 1$ hexane-EtOAc) of the residue first gave $20(120 \mathrm{mg}, 20 \%)$ and then $19(561 \mathrm{mg}, 61 \%) ;[\alpha]_{\mathrm{D}}-29^{\circ}(c 0.5)$, both isolated as a white foam. NMR data $\left(\mathrm{CDCl}_{3}\right)$ for 19: ${ }^{1} \mathrm{H}, \delta 7.83-6.73\left(\mathrm{~m}, 27 \mathrm{H}, 3 \mathrm{Ph}, \mathrm{MeOC}_{6} \mathrm{H}_{4} \mathrm{CH}_{2} \mathrm{O}\right.$, and $2 \mathrm{Phth}), 5.808\left(\mathrm{~m}, 1 \mathrm{H}, \mathrm{H}_{2} \mathrm{C}=\mathrm{CHCH}_{2} \mathrm{O}\right), 5.296\left(\mathrm{~d}, 1 \mathrm{H}, J_{1,2} 8.5 \mathrm{~Hz}, \mathrm{H}-1\right), 5.226$ and $5.152\left(2 \mathrm{~m}, 2 \mathrm{H}, \mathrm{H}_{2} \mathrm{C}=\mathrm{CHCH}_{2} \mathrm{O}\right), 5.158\left(\mathrm{~d}, 1 \mathrm{H}, J_{1^{\prime \prime}, 2^{\prime \prime \prime}} 2.3 \mathrm{~Hz}, \mathrm{H}-1^{\prime \prime \prime}\right), 5.098\left(\mathrm{t}, 1 \mathrm{H}, J_{4^{\prime \prime}, 5^{\prime \prime}}\right.$ $\left.9.9 \mathrm{~Hz}, \mathrm{H}-4^{\prime \prime}\right), 4.989$ and 4.947 (2 bt, each $\left.1 \mathrm{H}, \mathrm{H}-2^{\prime \prime \prime}, 3^{\prime \prime \prime}\right), 4.968$ and 4.545 ( $2 \mathrm{~d}$, each 1 $\mathrm{H}, \mathrm{PhCH}_{2} \mathrm{O}$ ) , $4.854\left(\mathrm{~d}, 1 \mathrm{H}, J_{1^{\prime}, 2^{\prime}} 8.5 \mathrm{~Hz}, \mathrm{H}-1^{\prime}\right), 4.851$ and $4.466\left(2 \mathrm{~d}\right.$, each $1 \mathrm{H}, \mathrm{PhCH}_{2} \mathrm{O}$ ), 4.757 ( $\left.\mathrm{m}, 1 \mathrm{H}, \mathrm{H}-4^{\prime \prime \prime}\right), 4.605$ ( $\left.\mathrm{s}, 1 \mathrm{H}, \mathrm{H}-1^{\prime \prime}\right), 3.803$ ( $\mathrm{s}, 3 \mathrm{H}, \mathrm{CH}_{3} \mathrm{OC}_{6} \mathrm{H}_{4} \mathrm{CH}_{2} \mathrm{O}$ ), 3.266 (s, $3 \mathrm{H}, \mathrm{CH}_{3} \mathrm{O}$ ), 3.173 ( dd, $\left.1 \mathrm{H}, J_{3^{\prime \prime}, 2^{\prime \prime}} 3.0, J_{3^{\prime \prime}, 4^{\prime \prime}} 9.9 \mathrm{~Hz}, \mathrm{H}-3^{\prime \prime}\right), 2.123,2.078,2.071,2.014$, and $1.887(5 \mathrm{~s}$, each $3 \mathrm{H}, 5 \Lambda \mathrm{c}) ;{ }^{13} \mathrm{C}, \delta 170.4,169.9,169.3(2 \mathrm{C})$, and $168.5\left(5 \mathrm{COCH}_{3}\right)$, 168.0 and 167.5 ( $C O$ Phth ), 158.8, 138.8, 137.6, 134.0, 133.3, 131.4, 131.1, 130.2, 128.8 126.4, 122.8, and 113.3 $\left(\mathrm{C}_{6} \mathrm{H}_{5} \mathrm{CH}_{2} \mathrm{O}, \mathrm{MeOC}_{6} \mathrm{H}_{4} \mathrm{CH}_{2} \mathrm{O}, \mathrm{H}_{2} \mathrm{C}=\mathrm{CHCH}_{2} \mathrm{O}\right.$, and Phth $), 116.4$ $\left(\mathrm{H}_{2} \mathrm{C}=\mathrm{CHCH}_{2} \mathrm{O}\right), 101.2,98.7,97.6$, and $96.7\left(\mathrm{C}-1,1^{\prime}, 1^{\prime \prime}, 1^{\prime \prime \prime}\right), 80.5,77.9,77.3,76.5,75.4$, 74.2 (2 C), 72.4, 71.9, 67.3 (2 C), 67.1, and 66.5 (C-3,4,5,3, $\left.4^{\prime}, 5^{\prime}, 2^{\prime \prime}, 3^{\prime \prime}, 4^{\prime \prime}, 5^{\prime \prime}, 2^{\prime \prime \prime}, 3^{\prime \prime \prime}, 4^{\prime \prime \prime}\right)$, $74.6,74.1,73.0,72.1,70.0,68.0,67.5,62.6$, and 58.4 (C-6,6 $, 6^{\prime \prime}, 5^{\prime \prime \prime}, 3 \mathrm{PhCH}_{2} \mathrm{O}$, $\mathrm{MeOC}_{6} \mathrm{H}_{4} \mathrm{CH}_{2} \mathrm{O}$, and $\left.\mathrm{H}_{2} \mathrm{C}=\mathrm{CHCH}_{2} \mathrm{O}\right), 56.3,56.1,55.3$, and 54.9 (C-2,2', $\mathrm{CH}_{3} \mathrm{OC}_{6} \mathrm{H}_{4} \mathrm{CH}_{2} \mathrm{O}$, and $\left.\mathrm{CH}_{3} \mathrm{O}\right), 20.7,20.6,20.5,20.4,20.3\left(5 \mathrm{COCH}_{3}\right)$. Anal. Calcd for $\mathrm{C}_{82} \mathrm{H}_{88} \mathrm{~N}_{2} \mathrm{O}_{28}: \mathrm{C}, 63.56 ; \mathrm{H}, 5.72$. Found: $\mathrm{C}, 63.19 ; \mathrm{H}, 5.83$. NMR data $\left(\mathrm{CDCl}_{3}\right)$ for $20:{ }^{1} \mathrm{H}$, $\delta 7.79-6.92(\mathrm{~m}, 14 \mathrm{H}, 2 \mathrm{Ph}$ and $\mathrm{Phth}), 6.592(\mathrm{~s}, 1 \mathrm{H}, \mathrm{H}-1), 5.842\left(\mathrm{~m}, 1 \mathrm{H}, \mathrm{H}_{2} \mathrm{C}=\mathrm{CHCH}_{2} \mathrm{O}\right)$, 5.265 and $5.176\left(2 \mathrm{~m}, 2 \mathrm{H}, \mathrm{H}_{2} \mathrm{C}=\mathrm{CHCH}_{2} \mathrm{O}\right), 5.261\left(\mathrm{~d}, 1 \mathrm{H}, J_{1^{\prime \prime} 2^{\prime \prime}} 3.5 \mathrm{~Hz}, \mathrm{H}-1^{\prime \prime}\right), 5.222$ (t, $\left.1 \mathrm{H}, J_{3^{\prime \prime}, \mathrm{z}^{\prime \prime}}=J_{3^{\prime \prime}, 4^{\prime \prime}}=5.0 \mathrm{~Hz}, \mathrm{H}-3^{\prime \prime}\right), 5.182\left(\mathrm{t}, 1 \mathrm{H}, J_{4^{\prime}, 3^{\prime}}=J_{4^{\prime}, 5^{\prime}}=9.7 \mathrm{~Hz}, \mathrm{H}-4^{\prime}\right), 5.047$ (dd, 1 $\left.\mathrm{H}, \mathrm{H}-2^{\prime \prime}\right), 4.818\left(\mathrm{~m}, 1 \mathrm{H}, \mathrm{H}-4^{\prime \prime}\right), 4.721\left(\mathrm{~s}, 1 \mathrm{H}, \mathrm{H}-1^{\prime}\right), 4.682$ and 4.407 ( $2 \mathrm{~d}$, each $1 \mathrm{H}$, $\mathrm{PhCH}_{2} \mathrm{O}$ ), 4.588 ( $\mathrm{s}, 2 \mathrm{H}, \mathrm{PhCH}_{2} \mathrm{O}$ ), 3.377 (dd, $1 \mathrm{H}, J_{3^{\prime}, 2^{\prime}} 2.9 \mathrm{~Hz}, \mathrm{H}-3$ ') $, 2.143,2.101$, $2.071,2.063$, and $1.949(5 \mathrm{~s}$, each $3 \mathrm{H}, 5 \mathrm{Ac}) ;{ }^{13} \mathrm{C}, \delta 170.5,169.6(2 \mathrm{C}), 169.3$, and 168.8 $\left(5 \mathrm{COCH}_{3}\right), 167.3$ ( $\mathrm{CO}$ Phth), 145.1 (C-1), 138.0, 137.6, 131.6, 128.2-126.9, 123.1 $\left(\mathrm{C}_{6} \mathrm{H}_{5} \mathrm{CH}_{2} \mathrm{O}, \mathrm{H}_{2} \mathrm{C}=\mathrm{CHCH}_{2} \mathrm{O}\right.$ and $\left.\mathrm{Phth}\right), 116.8\left(\mathrm{H}_{2} \mathrm{C}=\mathrm{CHCH}_{2} \mathrm{O}\right), 107.8(\mathrm{C}-2), 98.0(\mathrm{C}-$ $\left.1^{\prime}, 1^{\prime \prime}\right), 77.4(2 \mathrm{C}), 74.4,72.5,72.3,71.7,68.0,67.8(2 \mathrm{C})$, and 67.2 (C$\left.3,4,5,2^{\prime}, 3^{\prime}, 4^{\prime}, 5^{\prime}, 2^{\prime \prime}, 3^{\prime \prime}, 4^{\prime \prime}\right), 73.4,72.0,69.9,67.5,62.6$, and $59.0\left(\mathrm{C}-6,6^{\prime}, 5^{\prime \prime}, 2 \mathrm{PhCH}_{2} \mathrm{O}\right.$, and $\left.\mathrm{H}_{2} \mathrm{C}=\mathrm{CHCH}_{2} \mathrm{O}\right), 20.7(2 \mathrm{C}), 20.6(2 \mathrm{C})$, and $20.4\left(5 \mathrm{COCH}_{3}\right)$.

Methyl 3-O-benzyl-2-deoxy-4-O-\{3, 6-di-O-benzyl-2-deoxy-4-O-[4, 6-di-O-acetyl-3-Oallyl-2-O-(2,3,4-tri-O-acetyl- $\beta$-D-xylopyranosyl)- $\beta$-D-mannopyranosyl]-2-phthalimido$\beta$-D-glucopyranosyl $\}$-2-phthalimido- $\beta$-D-glucopyranoside (21).-To a solution of 19 (490 $\mathrm{mg}, 0.32 \mathrm{mmol})$ in $9: 1 \mathrm{MeCN}$-water $(10 \mathrm{~mL})$ was added ceric(IV) ammonium nitrate (380 mg, $0.69 \mathrm{mmol})$. When TLC (85:15 $\mathrm{CH}_{2} \mathrm{Cl}_{2}$-acetone) indicated the reaction to be complete $\left(1 \mathrm{~h} ; \mathbf{2 1}, R_{f} 0.35\right)$, the mixture was diluted with $\mathrm{CH}_{2} \mathrm{Cl}_{2}(500 \mathrm{~mL})$, washed with aq $5 \% \mathrm{NaHSO}_{3}(2 \times 50 \mathrm{~mL})$, aq $5 \% \mathrm{NaHCO}_{3}$, and water, dried $\left(\mathrm{MgSO}_{4}\right)$, filtered, and concentrated. Column chromatography of the residue ( $85: 15 \mathrm{CH}_{2} \mathrm{Cl}_{2}$-acetone) gave 21 , isolated as a white foam $(390 \mathrm{mg}, 86 \%) ;[\alpha]_{\mathrm{D}}-43^{\circ}(c 0.7) ; R_{f} 0.35$. NMR data $\left(\mathrm{CDCl}_{3}\right)$ : ${ }^{1} \mathrm{H}, \delta 7.846 .74(\mathrm{~m}, 23 \mathrm{H}, 3 \mathrm{Ph}$ and $2 \mathrm{Phth}), 5.801\left(\mathrm{~m}, 1 \mathrm{H}, \mathrm{H}_{2} \mathrm{C}=\mathrm{CHCH}_{2} \mathrm{O}\right.$ ), 5.359 (d, 1 $\left.\mathrm{H}, J_{1,2} 8.3 \mathrm{~Hz}, \mathrm{H}-1\right), 5.222$ and $5.147\left(2 \mathrm{~m}, 2 \mathrm{H}, H_{2} \mathrm{C}=\mathrm{CHCH}_{2} \mathrm{O}\right), 5.121\left(\mathrm{~d}, 1 \mathrm{H}, J_{1^{m}, 2^{m}} 2.9\right.$ $\left.\mathrm{Hz}, \mathrm{H}-1^{\prime \prime \prime}\right), 5.088$ ( t, $\left.1 \mathrm{H}, J_{4^{\prime \prime}, 5^{\prime \prime}} 9.9 \mathrm{~Hz}, \mathrm{H}-4^{\prime \prime}\right), 4.909$ (d, $1 \mathrm{H}, J_{1^{\prime}, 2^{\prime}}, 8.4 \mathrm{~Hz}, \mathrm{H}-1^{\prime}$ ) , 4.755 ( m, $\left.1 \mathrm{H}, \mathrm{H}-4^{\prime \prime \prime}\right), 4.593$ ( $\left.\mathrm{s}, 1 \mathrm{H}, \mathrm{H}-1^{\prime \prime}\right), 4.533$ (dd, $1 \mathrm{H}, J_{5^{\prime \prime \prime a x}, 5^{\prime \prime} e q}-13.1, J_{5^{\prime \prime \prime} a x / e q, 4^{\prime \prime}} 2.6 \mathrm{~Hz}, \mathrm{H}-$ $5^{\prime \prime \prime} a x$ or H-5"'eq), $3.271\left(\mathrm{~s}, 3 \mathrm{H}, \mathrm{CH}_{3} \mathrm{O}\right), 3.157\left(\mathrm{dd}, 1 \mathrm{H}, J_{3^{\prime \prime}, 2^{\prime \prime}} 3.0, J_{3^{\prime \prime}, 4^{\prime \prime}} 9.9 \mathrm{~Hz}, \mathrm{H}-3^{\prime \prime}\right)$, 
$2.132,2.126,2.067,2.015$, and $1.898(5 \mathrm{~s}$, each $3 \mathrm{H}, 5 \mathrm{Ac}) ;{ }^{13} \mathrm{C}, \delta 170.4,169.6,169.2(2$ C), $168.5\left(5 \mathrm{COCH}_{3}\right), 168.0$ and 167.4 ( $\mathrm{CO}$ Phth), 138.5, 138.2, 137.5, 133.9, 133.4, 131.3, 128.2-126.5, 123.0, and $122.8\left(\mathrm{C}_{6} \mathrm{H}_{5} \mathrm{CH}_{2} \mathrm{O}, \mathrm{H}_{2} \mathrm{C}=\mathrm{CHCH}_{2} \mathrm{O}\right.$, and Phth $), 116.4$ $\left(\mathrm{H}_{2} \mathrm{C}=\mathrm{CHCH}_{2} \mathrm{O}\right), \quad 101.2,98.8,97.6$, and $97.2 \quad\left(\mathrm{C}-1,1^{\prime}, 1^{\prime \prime}, 1^{\prime \prime \prime}\right), \quad 80.5, \quad 77.8$, $77.0,76.6,75.6,74.4,74.0,72.3,71.9,67.2(2 \mathrm{C}), 67.0$, and 66.4 (C$3,4,5,3^{\prime}, 4^{\prime}, 5^{\prime}, 2^{\prime \prime}, 3^{\prime \prime}, 4^{\prime \prime}, 5^{\prime \prime}, 2^{\prime \prime \prime}, 3^{\prime \prime \prime}, 4^{\prime \prime \prime}$ ), 74.2, 73.0 (2 C), 70.0, 68.2, 62.5, 60.5, and 58.4 (C$6,6^{\prime}, 6^{\prime \prime}, 5^{\prime \prime \prime}, 3 \mathrm{PhCH}_{2} \mathrm{O}$, and $\mathrm{H}_{2} \mathrm{C}=\mathrm{CHCH}_{2} \mathrm{O}$ ), 56.3, 56.1, and 55.3 (C-2,2' and $\mathrm{CH}_{3} \mathrm{O}$ ), 20.6 ( $2 \mathrm{C}), 20.4$, and $20.3(2 \mathrm{C})\left(5 \mathrm{COCH}_{3}\right)$. Anal. Calcd for $\mathrm{C}_{74} \mathrm{H}_{80} \mathrm{~N}_{2} \mathrm{O}_{27} \cdot \mathrm{H}_{2} \mathrm{O}: \mathrm{C}, 61.41 ; \mathrm{H}$, 5.71. Found: C, 61.40; H, 5.90 .

Methyl 3-O-benzyl-2-deoxy-4-O-\{3,6-di-O-benzyl-2-deoxy-4-O-[4,6-di-O-acetyl-3-Oallyl-2-O-(2, 3, 4-tri-O-acetyl- $\beta$-D-xylopyranosyl)- $\beta$-D-mannopyranosyl]-2-phthalimido$\beta$-D-glucopyranosyl\}-2-phthalimido-6-O-(2, 3, 4-tri-O-benzyl- $\alpha$-L-fucopyranosyl)- $\beta$-Dglucopyranoside (23).-A mixture of 21 (320 mg, $0.22 \mathrm{mmol}$ ) and ethyl 2,3,4-tri-Obenzyl-1-thio- $\beta$-L-fucopyranoside $[19](22 ; 160 \mathrm{mg}, 0.34 \mathrm{mmol})$ in $5: 1$ ether-1,2-dichloroethane $(6 \mathrm{~mL})$ containing powdered 4A molecular sieves ( $1 \mathrm{~g}$ ) was stirred for 30 min under $\mathrm{N}_{2}$. Then, iodonium dicollidine perchlorate [14] (310 $\mathrm{mg}, 0.66 \mathrm{mmol}$ ) was added and the mixture was stirred for $20 \mathrm{~min}$ at room temperature, when TLC ( $1: 1$ hexaneEtOAc) showed a complete conversion of $21\left(R_{f} 0.05\right)$ into two new compounds 23-a $\left(R_{f}\right.$ $0.11)$ and $23\left(R_{f} 0.25\right)$ as the minor and major product. The mixture was diluted with $\mathrm{CH}_{2} \mathrm{Cl}_{2}(250 \mathrm{~mL})$, filtered through Celite, washed with aq $10 \% \mathrm{Na}_{2} \mathrm{~S}_{2} \mathrm{O}_{3}(2 \times 25 \mathrm{~mL})$ and water, dried $\left(\mathrm{MgSO}_{4}\right)$, filtered, and concentrated. Column chromatography ( $1: 1$ hexaneEtOAc) of the residue first gave $23(203 \mathrm{mg}, 49 \%) ;[\alpha]_{\mathrm{D}}-40^{\circ}(c 1)$, and then 23-a (127 $\mathrm{mg}$ ); $[\alpha]_{\mathrm{D}}-41^{\circ}$ (c 1), both isolated as a white foam. NMR data $\left(\mathrm{CDCl}_{3}\right)$ for 23 : ${ }^{1} \mathrm{H}, \delta$ $7.82-6.73(\mathrm{~m}, 38 \mathrm{H}, 6 \mathrm{Ph}$ and $2 \mathrm{Phth}), 5.811\left(\mathrm{~m}, 1 \mathrm{H}, \mathrm{H}_{2} \mathrm{C}=\mathrm{CHCH}_{2} \mathrm{O}\right), 5.473(\mathrm{~d}, 1 \mathrm{H}$, $\left.J_{1,2} 8.5 \mathrm{~Hz}, \mathrm{H}-1\right), 5.226$ and $5.151\left(2 \mathrm{~m}, 2 \mathrm{H}, H_{2} \mathrm{C}=\mathrm{CHCH}_{2} \mathrm{O}\right), 5.078\left(\mathrm{t}, 1 \mathrm{H}, J_{4^{\prime \prime}, 5^{\prime \prime}} 9.8\right.$ $\mathrm{Hz}, \mathrm{H}-4^{\prime \prime \prime}$ ), 5.064 (d, $1 \mathrm{H}, J_{1^{\prime \prime \prime}, 2^{\prime \prime \prime}} 2.7 \mathrm{~Hz}, \mathrm{H}-1^{\prime \prime \prime}$ ), 4.846 ( d, $1 \mathrm{H}, J_{1^{\prime \prime}, 2^{\prime \prime}} 8.4 \mathrm{~Hz}, \mathrm{H}-1^{\prime \prime}$ ), 4.800 $\left(\mathrm{d}, 1 \mathrm{H}, J_{1^{\prime}, 2^{\prime}} 3.7 \mathrm{~Hz}, \mathrm{H}-1^{\prime}\right), 4.773$ and $4.701\left(2 \mathrm{~d}\right.$, each $\left.1 \mathrm{H}, \mathrm{PhCH} \mathrm{H}_{2} \mathrm{O}\right), 4.711(\mathrm{~m}, 1 \mathrm{H}, \mathrm{H}-$ $\left.4^{\prime \prime \prime \prime}\right), 4.582\left(\mathrm{~s}, 1 \mathrm{H}, \mathrm{H}-1^{\prime \prime \prime}\right), 4.420$ (dd, $1 \mathrm{H}, J_{5^{\prime \prime \prime \prime} e q / a x, 4^{\prime \prime \prime}} 3.3, J_{5^{\prime \prime \prime} a x, 5^{\prime \prime \prime} e q}-12.9 \mathrm{~Hz}, \mathrm{H}-5^{\prime \prime \prime \prime}$ ax or $\left.\mathrm{H}-5^{\prime \prime \prime \prime} e q\right), 3.186\left(\mathrm{~s}, 3 \mathrm{H}, \mathrm{CH}_{3} \mathrm{O}\right), 3.132$ (dd, $\left.1 \mathrm{H}, J_{3^{\prime \prime}, 2^{\prime \prime \prime}} 2.9, J_{3^{\prime \prime \prime}, 4^{\prime \prime \prime}} 9.9 \mathrm{~Hz}, \mathrm{H}-3^{\prime \prime \prime}\right), 2.086$, $2.035,2.010,1.907$, and $1.725(5 \mathrm{~s}$, each $3 \mathrm{H}, 5 \mathrm{Ac}), 1.020\left(\mathrm{~d}, 3 \mathrm{H}, J_{6^{\prime}, 5}, 6.5 \mathrm{~Hz}, \mathrm{H}-\right.$ $\left.6^{\prime}, 6^{\prime}, 6^{\prime}\right) ;{ }^{13} \mathrm{C}, \delta 170.5,169.6,169.3\left(2 \mathrm{C}\right.$ ), and $168.8\left(5 \mathrm{COCH}_{3}\right), 167.6$ (CO Phth), $138.7-138.4,137.6,134.0,133.5,133.3,131.4,131.1,128.3-127.1,126.6,126.5$, and $122.9\left(\mathrm{C}_{6} \mathrm{H}_{5} \mathrm{CH}_{2} \mathrm{O}, \mathrm{H}_{2} \mathrm{C}=\mathrm{CHCH}_{2} \mathrm{O}\right.$, and Phth $), 116.5\left(\mathrm{H}_{2} \mathrm{C}=\mathrm{CHCH}_{2} \mathrm{O}\right), 101.1,98.4,97.5$, and $96.6\left(\mathrm{C}-1,1^{\prime \prime}, 1^{\prime \prime \prime}, 1^{\prime \prime \prime \prime}\right), 96.6\left(\mathrm{C}-1^{\prime}, J_{\mathrm{C}-1^{\prime}, \mathrm{H}-1^{\prime}} 168.6 \mathrm{~Hz}\right), 80.2,79.2,78.0,77.3,76.9,76.4$, $75.3,74.9,73.8,73.6,72.0,71.8,67.7,67.3$ (2 C), 66.9, and 65.7 (C$\left.3,4,5,2^{\prime}, 3^{\prime}, 4^{\prime}, 5^{\prime}, 3^{\prime \prime}, 4^{\prime \prime}, 5^{\prime \prime}, 2^{\prime \prime \prime}, 3^{\prime \prime \prime}, 4^{\prime \prime \prime}, 5^{\prime \prime \prime}, 2^{\prime \prime \prime \prime}, 3^{\prime \prime \prime}, 4^{\prime \prime \prime}\right), 74.5,74.4$ ( 2 C), 73.0, 72.8, 72.3, 70.0, $68.0,63.8,62.6,58.7$ ( $\mathrm{C}-6,6^{\prime \prime}, 6^{\prime \prime \prime}, 5^{\prime \prime \prime}, 6 \mathrm{PhCH}_{2} \mathrm{O}$, and $\left.\mathrm{H}_{2} \mathrm{C}=\mathrm{CHCH}_{2} \mathrm{O}\right), 56.4,55.8$, and 55.5 $\left(\mathrm{C}-2,2^{\prime \prime}\right.$ and $\left.\mathrm{CH}_{3} \mathrm{O}\right), 20.7,20.6,20.4(2 \mathrm{C})$, and $20.1\left(5 \mathrm{COCH}_{3}\right), 16.2\left(\mathrm{C}-6^{\prime}\right)$.

Methyl 4-O-\{4-O-13-O-allyl-4-O-benzoyl-2-O-(2, 3, 4-tri-O-benzoyl- $\beta$-D-xylopyranosyl)- $\beta$-D-mannopyranosyl]-3, 6-di-O-benzyl-2-deoxy-2-phthalimido- $\beta$-D-glucopyrano$s y l\}$-3-O-benzyl-2-deoxy-2-phthalimido-6-O- $(2,3,4$-tri-O-benzyl- $\alpha$-L-fucopyranosyl)- $\beta$ $\mathrm{D}$-glucopyranoside (24).-To a solution of 23 (191 $\mathrm{mg}, 0.1 \mathrm{mmol})$ in $\mathrm{MeOH}(10 \mathrm{~mL})$ was added sodium methoxide until $\mathrm{pH}$ 9. The solution was stirred for $1 \mathrm{~h}$, when TLC (9:1 $\left.\mathrm{CH}_{2} \mathrm{Cl}_{2}-\mathrm{MeOH}\right)$ showed the $O$-deacetylation to be complete $\left(R_{f} 0.59\right)$, then neutralized with Dowex 50W $\left(\mathrm{H}^{+}\right)$resin, filtered, and concentrated. To a solution of the residue in 
pyridine ( $2 \mathrm{~mL}$ ) was added tert-butyldimethylsilyl chloride ( $47 \mathrm{mg}, 0.31 \mathrm{mmol}$ ), and the solution was stirred for $16 \mathrm{~h}$, when TLC (95:5 $\left.\mathrm{CH}_{2} \mathrm{Cl}_{2}-\mathrm{MeOH}\right)$ showed the silylation to be complete $(R, 0.53)$. Then benzoyl chloride $(100 \mu \mathrm{L}, 0.86 \mathrm{mmol})$ was added, and the mixture was stirred again for $16 \mathrm{~h}$, diluted with $\mathrm{CH}_{2} \mathrm{Cl}_{2}(250 \mathrm{~mL})$, washed with aq $5 \%$ $\mathrm{NaHCO}_{3}(25 \mathrm{~mL})$ and water, dried $\left(\mathrm{MgSO}_{4}\right)$, filtered, and concentrated. A solution of the residue and $p$-toluenesulfonic acid monohydrate $(99 \mathrm{mg}, 0.52 \mathrm{mmol})$ in $9: 1 \mathrm{MeCN}$-water $(7 \mathrm{~mL})$ was stirred for $30 \mathrm{~min}$ at room temperature. TLC ( $3: 1$ toluene-acetone) then showed a complete desilylation $\left(R_{f} 0.62\right)$. The solution was diluted with $\mathrm{CH}_{2} \mathrm{Cl}_{2}(250 \mathrm{~mL})$, washed with aq $5 \% \mathrm{NaHCO}_{3}(25 \mathrm{~mL})$ and water, dried $\left(\mathrm{MgSO}_{4}\right)$, filtered, and concentrated. Column chromatography ( $95: 5 \mathrm{CH}_{2} \mathrm{Cl}_{2}$-acetone) of the residue gave 24 , isolated as a syrup (152 mg, 72\%); $[\alpha]_{\mathrm{D}}-98^{\circ}(c 1) ; R_{f} 0.56$. NMR data $\left(\mathrm{CDCl}_{3}\right):{ }^{1} \mathrm{H}, \delta 8.18-6.65$ $(\mathrm{m}, 58 \mathrm{H}, 10 \mathrm{Ph}$ and $2 \mathrm{Phth}), 5.786\left(\mathrm{~m}, 1 \mathrm{H}, \mathrm{H}_{2} \mathrm{C}=\mathrm{CHCH}_{2} \mathrm{O}\right), 5.529,5.371$, and 5.143 (3 $\mathrm{m}$, each $\left.1 \mathrm{H}, \mathrm{H}-2^{\prime \prime \prime \prime}, 3^{\prime \prime \prime \prime}, 4^{\prime \prime \prime \prime}\right), 5.422\left(\mathrm{~d}, 1 \mathrm{H}, J_{1,2} 8.4 \mathrm{~Hz}, \mathrm{H}-1\right), 5.243(\mathrm{t}, 1 \mathrm{H}$, $\left.J_{4^{\prime \prime \prime}, 3^{\prime \prime \prime}}=J_{4^{\prime \prime \prime}, 5^{\prime \prime \prime}}=10.2 \mathrm{~Hz}, \mathrm{H}-4^{\prime \prime \prime}\right), 5.222$ and $5.094\left(2 \mathrm{~m}, 2 \mathrm{H}, \mathrm{H}_{2} \mathrm{C}=\mathrm{CHCH}_{2} \mathrm{O}\right), 4.987(\mathrm{~d}, 1$ $\left.\mathrm{H}, J_{1^{\prime \prime \prime}, 2^{\prime \prime \prime}} 2.2 \mathrm{~Hz}, \mathrm{H}-1^{\prime \prime \prime \prime}\right), 4.770$ and $4.671\left(2 \mathrm{~d}\right.$, each $\left.1 \mathrm{H}, \mathrm{PhCH}_{2} \mathrm{O}\right), 3.204\left(\mathrm{~s}, 3 \mathrm{H}, \mathrm{CH}_{3} \mathrm{O}\right)$, 1.082 (d, $\left.3 \mathrm{H}, J_{6^{\prime}, 5^{\prime}} 6.5 \mathrm{~Hz}, \mathrm{H}-6^{\prime}, 6^{\prime}, 6^{\prime}\right) ;{ }^{13} \mathrm{C}, \delta 168.1,167.7(2 \mathrm{C}$ ), and 165.5 (CO Phth), $165.5,165.1,164.8$, and $164.4\left(4 \mathrm{COC}_{6} \mathrm{H}_{5}\right), 116.9\left(\mathrm{H}_{2} \mathrm{C}=\mathrm{CHCH}_{2} \mathrm{O}\right), 100.0,98.5,98.3$, 97.1, and $96.5\left(\mathrm{C}-1,1^{\prime}, 1^{\prime \prime}, 1^{\prime \prime \prime}, 1^{\prime \prime \prime}\right), 56.7,55.9$, and $55.5\left(\mathrm{C}-2,2^{\prime \prime}\right.$ and $\left.\mathrm{CH}_{3} \mathrm{O}\right), 16.4\left(\mathrm{C}-6^{\prime}\right)$.

Methyl 4-O-\{4-O-13-O-allyl-4-O-benzoyl-6-O- $2,3,4,6-t e t r a-0-a c e t y l-\alpha-\mathrm{D}-m a n n o-$ pyranosyl)-2-O 528 points short- $(2,3,4$-tri-O-benzoyl- $\beta$-D-xylopyranosyl)- $\beta$-D-mannopyranosyl]-3, 6 -di-O-benzyl-2-deoxy-2-phthalimido- $\beta$-D-glucopyranosyl $\}-3-0$-benzyl-2deoxy-2-phthalimido-6-O-(2,3,4-tri-O-benzyl- $\alpha$-L-fucopyranosyl)- $\beta$-D-glucopyranoside (25).-A solution of 24 ( $100 \mathrm{mg}, 49 \mu \mathrm{mol})$ and 2,3,4,6-tetra- $O$-acetyl- $\alpha$-D-mannopyranosyl trichloroacetimidate [9] $(7 ; 57 \mathrm{mg}, 0.12 \mathrm{mmol})$ in $\mathrm{CH}_{2} \mathrm{Cl}_{2}(3 \mathrm{~mL})$ containing powdered $4 \mathrm{~A}$ molecular sieves $\left(200 \mathrm{mg}\right.$ ) was stirred under $\mathrm{N}_{2}$ for $30 \mathrm{~min}$. Trimethylsilyl triflate $(5 \mu \mathrm{L}, 26 \mu \mathrm{mol})$ was added at $-20^{\circ} \mathrm{C}$, and the mixture was stirred for $30 \mathrm{~min}$ at $0^{\circ} \mathrm{C}$. TLC (95:5 $\mathrm{CH}_{2} \mathrm{Cl}_{2}$-acetone) then showed the disappearance of $24\left(R_{f} 0.56\right)$ and the formation of a new product $25\left(R_{f} 0.46\right)$. Pyridine $(1 \mathrm{~mL})$ was added and the mixture was diluted with $\mathrm{CH}_{2} \mathrm{Cl}_{2}(250 \mathrm{~mL})$, filtered through Celite, washed with water, dried $\left(\mathrm{MgSO}_{4}\right)$, filtered, and concentrated. Column chromatography (95:5 $\mathrm{CH}_{2} \mathrm{Cl}_{2}$-acetone) of the residue gave 25, isolated as a syrup (53 mg, 45\%); $[\alpha]_{\mathrm{D}}-68^{\circ}(c 1)$. NMR data $\left(\mathrm{CDCl}_{3}\right):{ }^{1} \mathrm{H}, \delta$ 7.79-6.59 (m, 58 H, 10 Ph and $2 \mathrm{Phth}), 5.776\left(\mathrm{~m}, 1 \mathrm{H}, \mathrm{H}_{2} \mathrm{C}=\mathrm{CHCH}_{2} \mathrm{O}\right), 5.393(\mathrm{~d}, 1 \mathrm{H}$, $\left.J_{1,2} 8.5 \mathrm{~Hz}, \mathrm{H}-1\right), 5.346\left(\mathrm{t}, 1 \mathrm{H}, J_{4^{\prime \prime}, 3^{\prime \prime}}=J_{4^{\prime \prime}, 5^{\prime \prime}}=9.7 \mathrm{~Hz}, \mathrm{H}-4^{\prime \prime \prime}\right), 5.214(\mathrm{~m}, 1 \mathrm{H}$,

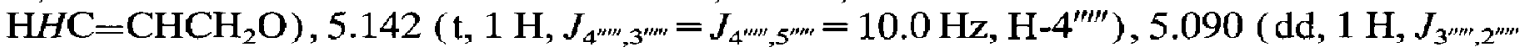
$\left.3.5 \mathrm{~Hz}, \mathrm{H}-3^{\prime \prime \prime \prime \prime}\right), 4.949$ (d, $1 \mathrm{H}, J_{1^{\prime}, 2^{\prime}} 3.1 \mathrm{~Hz}, \mathrm{H}-1^{\prime}$ ) , 4.781 (dd, $\left.1 \mathrm{H}, \mathrm{H}-2^{\prime \prime \prime \prime}\right), 4.762$ (s, $1 \mathrm{H}$,

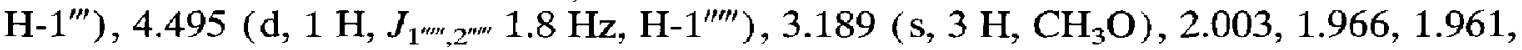
and $1.800(4 \mathrm{~s}$, each $3 \mathrm{H}, 4 \mathrm{Ac}), 1.059\left(\mathrm{~d}, 3 \mathrm{H}, J_{6^{\prime}, 5^{\prime}} 6.5 \mathrm{~Hz}, \mathrm{H}-6^{\prime}, 6^{\prime}, 6^{\prime}\right) ;{ }^{13} \mathrm{C}, \delta 170.5$, 169.8, 169.4, and 169.2 (4 $\left.\mathrm{COCH}_{3}\right), 168.1-167.3$ (CO Phth), 165.5, 165.3, 164.7, and $164.4\left(4 \mathrm{COC}_{6} \mathrm{H}_{5}\right), 117.1\left(\mathrm{H}_{2} \mathrm{C}=\mathrm{CHCH}_{2} \mathrm{O}\right), 100.1,98.5,98.2,97.3,97.1$, and $96.6(\mathrm{C}-$ $\left.1,1^{\prime}, 1^{\prime \prime}, 1^{\prime \prime \prime}, 1^{\prime \prime \prime \prime}, 1^{\prime \prime \prime \prime}\right), 56.6,55.9$, and $55.5\left(\mathrm{C}-2,2^{\prime \prime}\right.$ and $\left.\mathrm{CH}_{3} \mathrm{O}\right), 20.6-20.4\left(\mathrm{COCH}_{3}\right), 16.4$ $\left(\mathrm{C}-6^{\prime}\right)$.

Methyl 4-O-\{4-O-[4-O-benzoyl-6-O- $(2,3,4,6$-tetra-O-acetyl- $\alpha$-D-mannopyranosyl)2 -O-(2,3, 4-tri-O-benzoyl- $\beta$-D-xylopyranosyl)- $\beta$-D-mannopyranosyl]-3, 6-di-O-benzyl-2deoxy-2-phthalimido- $\beta$-D-glucopyranosyl $\}-3-\mathrm{O}$-benzyl-2-deoxy-2-phthalimido-6-O- $(2,3$, 4-tri-O-benzyl- $\alpha$-L-fucopyranosyl)- $\beta$-D-glucopyranoside (26).-A solution of 25 ( $53 \mathrm{mg}$, 
$22 \mu \mathrm{mol}$ ), tris (triphenylphosphine) rhodium( I) chloride (13 mg, $15 \mu \mathrm{mol}$ ), and 1,4-diazabicyclo [2.2.2] octane ( $2 \mathrm{mg}, 18 \mu \mathrm{mol}$ ) in 7:3:1 EtOH-toluene-water $(3 \mathrm{~mL})$ was boiled under reflux for $2.5 \mathrm{~h}$, then cooled, and concentrated. A solution of the residue in 9:1 acetone-M aq $\mathrm{HCl}(5 \mathrm{~mL})$ was then boiled under reflux for $30 \mathrm{~min}$, when TLC (95:5 $\mathrm{CH}_{2} \mathrm{Cl}_{2}$-acetone) indicated a complete conversion of 25 into $26\left(R_{f} 0.36\right)$. The solution was concentrated, and a solution of the residue in $\mathrm{CH}_{2} \mathrm{Cl}_{2}(100 \mathrm{~mL})$ was washed with aq $5 \% \mathrm{NaHCO}_{3}(10 \mathrm{~mL})$ and water, dried $\left(\mathrm{MgSO}_{4}\right)$, filtered, and concentrated. Preparative TLC (95:5 $\mathrm{CH}_{2} \mathrm{Cl}_{2}$-acetone) of the residue gave 26, isolated as a syrup ( $38 \mathrm{mg}$, 73\%); $[\alpha]_{\mathrm{D}}-23^{\circ}(c 1) ; R_{f}$ 0.36. NMR data $\left(\mathrm{CDCl}_{3}\right):{ }^{1} \mathrm{H}, \delta 7.94-6.73(\mathrm{~m}, 58 \mathrm{H}, 10 \mathrm{Ph}$ and 2 Phth), 5.630 (t, $\left.1 \mathrm{H}, J_{3^{\prime \prime \prime}, 4^{\prime \prime \prime}} 7.3 \mathrm{~Hz}, \mathrm{H}-3^{\prime \prime \prime \prime}\right), 5.461$ (dd, $\left.1 \mathrm{H}, J_{2^{\prime \prime \prime \prime}, 3^{\prime \prime \prime}} 7.5 \mathrm{~Hz}, \mathrm{H}-2^{\prime \prime \prime \prime}\right), 5.027$ (d, $\left.1 \mathrm{H}, J_{1^{\prime \prime \prime \prime}, 2^{\prime \prime \prime \prime}} 5.4 \mathrm{~Hz}, \mathrm{H}-1^{\prime \prime \prime \prime}\right), 4.954\left(\mathrm{dd}, 1 \mathrm{H}, J_{2^{\prime \prime \prime}, 1^{\prime \prime \prime \prime}} 1.9 \mathrm{~Hz}, J_{2^{\prime \prime \prime}, 3^{\prime \prime \prime \prime}} 2.7 \mathrm{~Hz}, \mathrm{H}-2^{\prime \prime \prime \prime}\right), 3.174(\mathrm{~s}, 3$ $\mathrm{H}, \mathrm{CH}_{3} \mathrm{O}$ ), $1.976,1.971,1.929$, and 1.842 ( $4 \mathrm{~s}$, each $3 \mathrm{H}, 4 \mathrm{Ac}$ ), 1.039 (d, $3 \mathrm{H}, J_{6}, 5,6.5$ $\left.\mathrm{Hz}, \mathrm{H}-6^{\prime}, 6^{\prime}, 6^{\prime}\right)$.

Methyl 4-O-\{4-0-[4-O-benzoyl-3,6-di-O-(2,3,4,6-tetra-O-acetyl- $\alpha-\mathrm{D}-$ mannopyranosyl)-2-O-(2, 3, 4-tri-O-benzoyl- $\beta$-D-xylopyranosyl $)-\beta$-D-mannopyranosyl $]-3,6-d i-\mathrm{O}-$ benzyl-2-deoxy-2-phthalimido- $\beta$-D-glucopyranosyl $\}-3-\mathrm{O}-$ benzyl-2-deoxy-2-phthalimido-6-O(2,3, 4-tri-O-benzyl- $\alpha$-L-fucopyranosyl)- $\beta$-D-glucopyranoside (27). - A solution of 26 (37 $\mathrm{mg}, 16 \mu \mathrm{mol})$ and 2,3,4,6-tetra- $O$-acetyl- $\alpha$-D-mannopyranosyl trichloroacetimidate [9] (7; $25 \mathrm{mg}, 50 \mu \mathrm{mol})$ in $\mathrm{CH}_{2} \mathrm{Cl}_{2}(2 \mathrm{~mL})$ containing powdered 4A molecular sieves (100 $\mathrm{mg}$ ) was stirred under $\mathrm{N}_{2}$ for $30 \mathrm{~min}$. Trimethylsilyl triflate ( $3 \mu \mathrm{L}, 17 \mu \mathrm{mol}$ ) was added at $-20^{\circ} \mathrm{C}$, and the mixture was stirred for $2 \mathrm{~h}$ at $-10^{\circ} \mathrm{C}$. TLC $\left(37: 3 \mathrm{CH}_{2} \mathrm{Cl}_{2}-\right.$ acetone $)$ then showed the disappearance of $26\left(R_{f} 0.71\right)$, and a new product $27\left(R_{f} 0.58\right)$. Pyridine ( 1 $\mathrm{mL})$ was added, and the mixture was diluted with $\mathrm{CH}_{2} \mathrm{Cl}_{2}(100 \mathrm{~mL})$, filtered through Celite, washed with water, dried $\left(\mathrm{MgSO}_{4}\right)$, filtered, and concentrated. Preparative TLC (4:1 toluene-acetone) of the residue gave 27 , isolated as a syrup $(38 \mathrm{mg}, 88 \%) ;[\alpha]_{\mathrm{D}}-45^{\circ}(c$ 1, EtOAc) ; $R_{f}$ 0.38. NMR data $\left(\mathrm{CDCl}_{3}\right):{ }^{1} \mathrm{H}, \delta 8.11-6.64(\mathrm{~m}, 58 \mathrm{H}, 10 \mathrm{Ph}$ and $2 \mathrm{Phth})$, $3.173\left(\mathrm{~s}, 3 \mathrm{H}, \mathrm{CH}_{3} \mathrm{O}\right), 2.048,2.016,1.999,1.968,1.953,1.849,1.825$, and 1.801 ( $8 \mathrm{~s}$, each $3 \mathrm{H}, 8 \mathrm{Ac}$ ), 1.036 (d, $3 \mathrm{H}, J_{6^{\prime}, 5^{\prime}} 6.5 \mathrm{~Hz}, \mathrm{H}-66^{\prime}, 6^{\prime}, 6^{\prime}$ ); ${ }^{13} \mathrm{C}, \delta 100.5,99.1,98.5$ (2 C), 97.3, and $97.0(2 \mathrm{C})\left(\mathrm{C}-1,1^{\prime}, 1^{\prime \prime}, 1^{\prime \prime \prime}, 1^{\prime \prime \prime}, 1^{\prime \prime \prime}, 1^{\prime \prime \prime \prime}\right), 56.6,55.9$, and $55.5\left(\mathrm{C}-2,2^{\prime \prime}\right.$ and $\left.\mathrm{CH}_{3} \mathrm{O}\right), 20.6-$ $20.2\left(\mathrm{COCH}_{3}\right), 16.4\left(\mathrm{C}-6^{\prime}\right)$.

Methyl 2-acetamido-4-O-[2-acetamido-2-deoxy-4-O-(3,6-di-O- $\alpha$-D-mannopyranosyl2-O- $\beta$-D-xylopyranosyl- $\beta$-D-mannopyranosyl)- $\beta$-D-glucopyranosyl]-2-deoxy-6-O- $\alpha-\mathrm{I}$ fucopyranosyl- $\beta$-D-glucopyranoside (5).-A solution of 27 ( $35 \mathrm{mg}, 13 \mu \mathrm{mol}$ ) and hydrazine acetate $(260 \mathrm{mg}, 2.8 \mathrm{mmol})$ in $\mathrm{EtOH}(7.5 \mathrm{~mL})$ was boiled under reflux for $16 \mathrm{~h}$, then cooled, and concentrated. A solution of the residue in $1: 1$ pyridine- $\mathrm{Ac} \mathrm{c}_{2} \mathrm{O}(4 \mathrm{~mL})$ was stirred for $16 \mathrm{~h}$ at room temperature, then concentrated, and toluene, $\mathrm{EtOH}$, and $\mathrm{CH}_{2} \mathrm{Cl}_{2}$ (each $3 \times 10 \mathrm{~mL}$ ) were evaporated from the residue. Column chromatography of the residue on Sephadex LH-20 (1:1 MeOH-CH $\left.\mathrm{Cl}_{2}\right)$ afforded a white solid. To a solution of this product in 1:1 EtOH-EtOAc $(5 \mathrm{~mL})$, containing AcOH $(50 \mu \mathrm{L})$, was added $10 \% \mathrm{Pd}-\mathrm{C}$ ( $50 \mathrm{mg}$ ), and hydrogenolysis was performed at atmospheric pressure for $24 \mathrm{~h}$. Then the mixture was filtered through Celite and concentrated, and toluene, EtOH, and $\mathrm{CH}_{2} \mathrm{Cl}_{2}$ (each $3 \times 5 \mathrm{~mL}$ ) were evaporated from the residue. Because ${ }^{1} \mathrm{H}$ NMR analysis showed that the dephthaloylation was incomplete, the residue was treated with $\mathrm{MeNH}_{2}-\mathrm{EtOH}(33 \%, 15$ $\mathrm{mL}$ ) for $24 \mathrm{~h}$, concentrated, and treated with $\mathrm{Ac}_{2} \mathrm{O}(300 \mu \mathrm{L})$ in $\mathrm{MeOH}$ ( $3 \mathrm{~mL}$ ) for $24 \mathrm{~h}$. The solution was concentrated, and toluene, $\mathrm{EtOH}$, and $\mathrm{CH}_{2} \mathrm{Cl}_{2}$ (each $3 \times 10 \mathrm{~mL}$ ) were 
evaporated from the residue, which was fractionated on Sephadex LH-20 (1:1 MeOH$\mathrm{CH}_{2} \mathrm{Cl}_{2}$ ) to yield impure 5. This product was then subjected to purification by RP-HPLC on a $\mathrm{Cp}$ tm Spher C18 column (250×4.6 mm, Chrompack) by elution with 95:5 water$\mathrm{MeOH}$ for $5 \mathrm{~min}$, followed by a linear gradient of 95:5 $\rightarrow 1: 1$ water-MeOH for $35 \mathrm{~min}$, and detection at $205 \mathrm{~nm}$. The fraction eluting at $14.3 \mathrm{~min}$ was collected and concentrated to yield 5 , isolated as a white amorphous solid $(13 \mathrm{mg}, 83 \%) ;[\alpha]_{\mathrm{D}}-24^{\circ}\left(c 0.1, \mathrm{H}_{2} \mathrm{O}\right)$. For ${ }^{1}$ H NMR data, see Table 1.

\section{Acknowledgements}

This investigation was supported by the Netherlands Foundation for Chemical Research (SON) with financial aid from the Netherlands Organization for Scientific Research (NWO), including an NWO visitors grant (J.K.). We thank Drs. B.A. Spronk, C.H. Hokke, and J.P.M. Lommerse for the recording of the 500-MHz ${ }^{1} \mathrm{H}$ NMR spectra, and Dr. A.M.P. van Steijn for fruitful discussions.

\section{References}

[1] J.P. Kamerling, Pure Appl. Chem., 63 (1991) 465-472.

[2] J.A. van Kuik, H. van Halbeek, J.P. Kamerling, and J.F.G. Vliegenthart, J. Biol. Chem., 260 ( 1985 ) 13984 13988.

[3] B. Fournet, Y. Leroy, J.-M. Wieruszeski, J. Montreuil, R.D. Poretz, and R. Goldberg, Eur. J. Biochem., 166 (1987) 321-324.

[4] D. Ashford, R.A. Dwek, J.K. Welply, S. Amatayakul, S.W. Homans, H. Lis, G.N. Taylor, N. Sharon, and T.W. Rademacher, Eur. J. Biochem., 166 (1987) 311-320.

[5] J.B. Bouwstra, J. Kerékgyártó, J.P. Kamerling, and J.F.G. Vliegenthart, Carbohydr. Res., 186 (1989) 3949.

[6] J.B. Bouwstra, E.C. Spoelstra, P. de Waard, B.R. Leeflang, J.P. Kamerling, and J.F.G. Vliegenthart, Eur. J. Biochem., 190 (1990) 113-122.

[7] B.R. Leeflang, J.B. Bouwstra, J. Kerékgyártó, J.P. Kamerling, and J.F.G. Vliegenthart, Carbohydr. Res., 208 (1990) 117-126.

[8] H. Mulder, H. Schachter, M. de Jong-Brink, J.G.M. van der Ven, J.P. Kamerling, and J.F.G. Vliegenthart, Eur. J. Biochem., 210 (1991) 459-465.

[9] J. Kerékgyártó, J.P. Kamerling, J.B. Bouwstra, J.F.G. Vliegenthart, and A. Lipták, Carbohydr. Res., 186 (1989) 51-62.

[10] J. Kerékgyártó, J.G.M. van der Ven, J.P. Kamerling, A. Lipták, and J.F.G. Vliegenthart, Carbohydr. Res., 238 (1993) 135-145.

[11] J.G.M. van der Ven, J.C.H.M. Wijkmans, J.P. Kamerling, and J.F.G. Vliegenthart, Carbohydr. Res., 253 (1994) 121-139.

[12] J. Kerékgyártó, J.G.M. van der Ven, J.P. Kamerling, A. Lipták, and J.F.G. Vliegenthart, Abstr. Eur. Carbohydr. Symp., 5th, Prague, Czechoslovakia, 1989, A64.

[13] A.M.P. van Steijn, Ph.D. Thesis, Utrecht University, The Netherlands, 1990.

[14] G. Veeneman, Ph.D. Thesis, State University of Leiden, The Netherlands, 1991.

[15] B. Classon, P.J. Garegg, S. Oscarson, and A.K. Tidén, Carbohydr. Res., 216 (1991) 187-196.

[16] E.J. Corey and J.W. Suggs, J. Org. Chem., 38 (1973) 3224.

[17] M.S. Motawia, J. Wengel, A.E.S. Abdel-Megid, and E.B. Pedersen, Synthesis, (1989) 384.

[18] D.A. Schwartz, H.H. Lee, J.P. Carver, and J. Krepinsky, Can. J. Chem., 63 (1985) 1073-1079.

[19] H. Lönn, Carbohydr. Res., 139 ( 1985) 105-113. 
[20] II. Lönn, Carbohydr. Res., 139 (1985) 115-121.

[21] B. Classon, P.J. Garegg, and B. Samuelsson, Acta Chem. Scand., Ser. B, 38 (1984) 419-422.

[22] A. Sturm, J.A. van Kuik, J.F.G. Vliegenthart, and M.J. Chrispeels, J. Biol. Chem., 262 (1987) 13392-13403.

[23] G. d'Andrea, J.B. Bouwstra, J.P. Kamerling, and J.F.G. Vliegenthart, Glycoconjugate J., 5 (1988) 151157. 